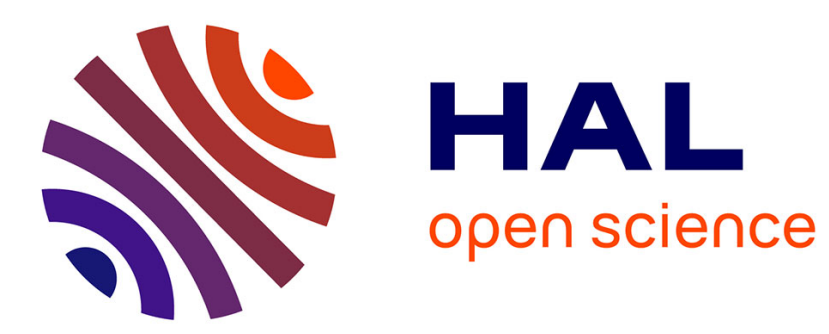

\title{
Subcarrier and Power Allocation for the Downlink of Multicarrier NOMA Systems
}

Yaru Fu, Lou Salaun, Chi Wan Sung, Chung Shue Chen

\section{To cite this version:}

Yaru Fu, Lou Salaun, Chi Wan Sung, Chung Shue Chen. Subcarrier and Power Allocation for the Downlink of Multicarrier NOMA Systems. IEEE Transactions on Vehicular Technology, In press, 67 (12), pp.11833-11847. 10.1109/TVT.2018.2875601 . hal-01887203

\section{HAL Id: hal-01887203 https://hal.inria.fr/hal-01887203}

Submitted on 3 Oct 2018

HAL is a multi-disciplinary open access archive for the deposit and dissemination of scientific research documents, whether they are published or not. The documents may come from teaching and research institutions in France or abroad, or from public or private research centers.
L'archive ouverte pluridisciplinaire HAL, est destinée au dépôt et à la diffusion de documents scientifiques de niveau recherche, publiés ou non, émanant des établissements d'enseignement et de recherche français ou étrangers, des laboratoires publics ou privés. 


\title{
Subcarrier and Power Allocation for the Downlink of Multicarrier NOMA Systems
}

\author{
Yaru Fu, Member, IEEE, Lou Salaün, Student Member, IEEE, \\ Chi Wan Sung, Senior Member, IEEE and Chung Shue Chen, Senior Member, IEEE
}

\begin{abstract}
This paper investigates the joint subcarrier and power allocation problem for the downlink of a multi-carrier non-orthogonal multiple access (MC-NOMA) system. A novel three-step resource allocation framework is designed to deal with the sum rate maximization problem. In Step 1, we relax the problem by assuming each of the users can use all subcarriers simultaneously. With this assumption, we prove the convexity of the resultant power control problem and solve it via convex programming tools to get a power vector for each user; In Step 2, we allocate subcarriers to users by a heuristic greedy manner with the obtained power vectors in Step 1; In Step 3, the proposed power control schemes used in Step 1 are applied once more to further improve the system performance with the obtained subcarrier assignment of Step 2. To solve the maximization problem with fixed subcarrier assignments in both Step 1 and Step 3, a centralized power allocation method based on projected gradient descent algorithm and two distributed power control strategies based respectively on pseudo-gradient algorithm and iterative waterfilling algorithm are investigated. Numerical results show that our proposed three-step resource allocation algorithm could achieve comparable sum rate performance to the existing nearoptimal solution with much lower computational complexity and outperforms power controlled OMA scheme. Besides, a tradeoff between user fairness and sum rate performance can be achieved via applying different user power constraint strategies in the proposed algorithm.
\end{abstract}

Index Terms-Multi-carrier non-orthogonal multiple access (MC-NOMA), successive interference cancellation (SIC), game theory, Nash equilibrium, gradient and pseudo-gradient descent, synchronous iterative waterfilling algorithm (SIWA).

\section{INTRODUCTION}

In the third generation partnership project Long Term Evolution (3GPP-LTE) and LTE-Advanced (LTE-A) cellular networks, orthogonal frequency division multiple access (OFDMA) has been widely applied, in which the whole system bandwidth is divided into multiple orthogonal subcarriers and each subcarrier is exclusively utilized by at most one user during each time slot at each base station (BS). Therefore,

Copyright (c) 2015 IEEE. Personal use of this material is permitted. However, permission to use this material for any other purposes must be obtained from the IEEE by sending a request to pubs-permissions@ieee.org.

Y. Fu and C. W. Sung are with the Dept. of Electronic Engineering, City University of Hong Kong, Tat Chee Avenue, Kowloon, Hong Kong SAR (email: yarufu2-c@my.cityu.edu.hk; albert.sung@cityu.edu.hk).

L. Salaün and C. S. Chen are with Bell Labs, Nokia ParisSaclay, 91620 Nozay, France (e-mail: lou.salaun@nokia-bell-labs.com; chung_shue.chen@nokia-bell-labs.com).

This work was partially supported by a grant from the Research Grants Council of the Hong Kong Special Administrative Region, China (Project No. CityU 11216416). A part of the work was carried out at LINCS (www.lincs.fr), and presented at IEEE International Conference on Communications (ICC), May, Paris, 2017 [1].
OFDMA can avoid intra-cell interference by performing user transmission scheduling at the BS. Moreover, it can be implemented with low-complexity receivers. However, the spectral efficiency is in general under-utilized due to the requirement of channel access orthogonality.

The mobile data traffic for cellular systems is expected to increase by 1000 folds by 2020, seeking efficient multiple access technologies becomes very important, as it is the key for meeting the dramatically increasing bandwidth demand. Nonorthogonal multiple access (NOMA) has recently received significant attention and has been regarded as a promising candidate for the fifth generation $(5 \mathrm{G})$ cellular systems. In contrast to orthogonal multiple access (OMA), NOMA allows the multiplexing of multiple users with different power levels on the same frequency resource block, which provides a higher system spectral efficiency [2], [3].

Since several users are multiplexed on the same subcarrier simultaneously, successive interference cancellation (SIC) is adopted at the receiver side to mitigate the resulting co-channel interference. Therefore, power must be allocated properly among the multiplexed users on each subcarrier such that interfering signals can be correctly decoded and subtracted from the received signal of some users. Fractional transmit power control (FTPC) is a sub-optimal but commonly used power control strategy for users sum rate maximization problems, which allocates power according to the individual link condition of each user [2], [4]. In [5], power allocation in single-cell NOMA system is performed in a fixed manner. In [6] and [7], distributed power allocation algorithms are proposed to minimize total power consumption subject to user data rate constraints for the downlink and uplink multi-cell NOMA systems, respectively. In addition, there exists some other works that investigate the power control for NOMA in heterogeneous networks [8], cooperative systems [9], [10] and multi-antenna scenarios [11], [12]. Except power control, fairness is also an important feature of NOMA. In [13], the resource allocation fairness between NOMA and OMA schemes is compared, based on which a hybrid NOMA-OMA scheme is proposed to further enhance the users fairness. Recent works [14], [15] investigate NOMA with two sets of orthogonal signal waveforms, in which power imbalance between different users' signals is not needed. Moreover, deep learning has been applied in NOMA to estimate the channel state information by learning the environment automatically via offline learning [16].

For multicarrier NOMA (MC-NOMA) systems, subcarrier assignment and power control for multiplexed users are two 
interacted factors for achieving high system performance. The work in [17] demonstrates that in practical LTE cellular systems, MC-NOMA has better system-level downlink performance in terms of user throughput than that of OFDMA networks by realistic computer simulation. In [18], a monotonic optimization method is proposed to achieve the optimal subcarrier and power allocation for MC-NOMA system. However, it is assumed that each subcarrier can accommodate at most two users, which restricts the application of the designed algorithm. The authors of [19] propose a low-complexity algorithm of jointly optimizing subcarrier assignment and power allocation to minimize the total transmit power of OFDMbased NOMA network. In addition, the energy-efficient resource allocation problems for MC-NOMA systems with perfect channel state information (CSI) and imperfect CSI are investigated in [20] and [21], respectively. An iterative algorithm is provided in [22] to solve the sub-channel and power allocation problem in MC-NOMA network. The designed resource allocation strategy is not so efficient as swapmatching is adopted to do the subcarrier assignment during each iteration, especially when the number of user is large. In [23], a greedy user selection and sub-optimal power allocation scheme based on difference-of-convex (DC) programming is presented to maximize the weighted throughput in a twouser NOMA system. It is observable that the scheme has high computational complexity due to the DC programming in optimizing the power allocation among different subcarriers and different users. In [24], various user pairing algorithms for multi-input and single-output (MISO) MC-NOMA system are proposed. However, the performance gain of [24] is limited due to the use of naive power control schemes such as fixed power allocation (FPA) and FTPC among multiplexed users. Besides, a joint power and channel allocation problem for sum rate maximization of MC-NOMA system is formulated in [25], which is shown NP-hard and solved by Lagrangian duality and dynamic programming (LDDP). To gauge the performance of LDDP, the authors investigate the optimality bound on the global optimum and show that LDDP could achieve a closeto-upper-bound performance.

Motivated by the aforementioned observations and the much lower latency requirement of $5 \mathrm{G}$, we propose a timeefficient joint subcarrier and power allocation algorithm for MC-NOMA, which could achieve comparable performance to LDDP. Our main contributions are summarized as follows:

1) A resource allocation framework is designed, which consists of the following three steps:

Step 1: Assume each user can use all subcarriers. By this assumption, the problem becomes convex and can be solved efficiently. A centralized or some distributed algorithms for the resultant power control subproblem are designed.

Step 2: Based on the power allocation solution obtained, the subcarrier assignment problem is solved, taking into account practical system constraints.

Step 3: Given the above subcarrier allocation, Step 3 solves a convex problem, i.e., the power allocation is further adjusted to optimize the final system performance.

The above 3-step methodology allows us to solve the problem efficiently. This is how we handle the problem, why we do 3 steps. And then, we show its near optimal performance by comprehensive simulation results.

2) In Steps 1 and 3, the subcarrier assignment is fixed. We prove that the resultant subproblem is convex, which can be optimally solved in a centralized manner via gradient descent algorithm. Alternatively, it can be solved more efficiently in a distributed manner. To do this, we model the problem as a multi-player concave game, and prove the existence and uniqueness of its Nash equilibrium. Two distributed algorithms, namely, pseudo-gradient descent and iterative waterfilling, are investigated, and their convergence properties are established. Furthermore, we analyze the asymptotic computational complexity of the proposed power control schemes. Except for the abovementioned, in Step 2, a heuristic method, which makes use of the result obtained in Step 1, is proposed for subcarrier assignment.

3) Under our framework, different allocation algorithms can be applied to solve each subproblem in the three steps. Numerical results show that the algorithms constructed under this framework, could generally achieve comparable performance to LDDP with much fewer computation steps and outperform standard power-controlled OMA scheme in terms of sum rate performance. Different tradeoffs can be achieved by using different algorithms in the three steps. Furthermore, a balancing between sumrate performance and user fairness can also be obtained by adopting different user power budget strategies.

The rest of this paper is organized as follows. In Section II, we present the system model and formulate the sum rate maximization problem mathematically. In Section III, the proposed three-step resource allocation framework is introduced. The centralized gradient descent algorithm and some distributed power control methods are analyzed in Section IV and Section V, respectively. In Section VI, we evaluate the performance of our proposed three-step subcarrier and power allocation algorithms and some other existing resource allocation schemes through computer simulations. Finally, the pros and cons of the designed resource allocation schemes and the future work are presented in Section VII.

\section{System Model AND Problem Formulation}

\section{A. System Model}

This section describes the system model and the problem to be solved in this paper. The downlink of a multi-user MC-NOMA system with one base station (BS) serving $K$ users is considered. Denote the set of all users' indices by $\mathcal{K} \triangleq\{1,2, \ldots, K\}$. The overall system bandwidth $W$ is divided into $N$ subcarriers. We denote the index set of these $N$ subcarriers by $\mathcal{N} \triangleq\{1,2, \ldots, N\}$. For $n \in \mathcal{N}$, let $W_{n}$ be the bandwidth of subcarrier $n$, where $\sum_{n \in \mathcal{N}} W_{n}=W$. Assume there is no interference among different subcarriers because of the orthogonal frequency division. 
For $k \in \mathcal{K}$ and $n \in \mathcal{N}$, let $g_{k}^{n}$ be the link gain of user $k$ on subcarrier $n$. We assume a frequency-flat block fading channel for each subcarrier. Let $p_{k}^{n} \geq 0$ be the allocated transmit power to user $k$ on subcarrier $n$. User $k$ is said to be multiplexed on subcarrier $n$ if $p_{k}^{n}>0$. Additionally, we assume the BS has a total power budget, which is denoted by $P_{\text {total }}$. Moreover, we set maximum power allocation for each user, i.e., $\sum_{n=1}^{N} p_{k}^{n} \leq \bar{p}_{k}$, where $\bar{p}_{k}>0$ and $k \in \mathcal{K}$, to ensure the effort fairness among users [25]-[27]. Note that the maximum power budget for each user can be set arbitrarily for resource allocation fairness or any preference, subject to the constraint $\sum_{k=1}^{K} \bar{p}_{k} \leq P_{\text {total }}$. Furthermore, denote by $\eta_{k}^{n}$ the received noise power of user $k$ on subcarrier $n$. For notation simplicity, we normalize the noise power as $\tilde{\eta}_{k}^{n} \triangleq \eta_{k}^{n} / g_{k}^{n}$.

We consider the scenario in which BS allocates subcarriers to its attached users and multiplexes them on a given subcarrier using superposition coding. For $n \in \mathcal{N}$, let $\mathcal{U}_{n}$ be the set of users to whom subcarrier $n$ is assigned. Therefore, each subcarrier can be modeled as a multi-user Gaussian broadcast channel and SIC is applied at the receiver side when it is possible to eliminate the intra-band interference.

Since SIC is applied, we need to consider the decoding order of users on the same subcarrier. For $n \in \mathcal{N}$, let $\Pi_{n}$ be the set of all possible permutations of $\mathcal{U}_{n}$. For example, if users $u, v \in \mathcal{K}$ are multiplexed on subcarrier $n$, i.e., $\mathcal{U}_{n}=\{u, v\}$, then

$$
\Pi_{n}=\{(u, v),(v, u)\} .
$$

Let $\boldsymbol{\pi}_{n} \in \Pi_{n}$ be the decoding order of the users on subcarrier $n$. Let $\pi_{n}(i)$, where $i \in\left\{1,2, \ldots,\left|\mathcal{U}_{n}\right|\right\}$, be its $i$-th component, which means that user $\pi_{n}(i)$ first decodes the signals of $\pi_{n}(1)$ to $\pi_{n}(i-1)$, then subtracts these signals, and finally decodes its intended message by treating the signals of the remaining users on subcarrier $n$ as noise. Note that $\pi_{n}$ can be seen as a vector function of the normalized noise power of each multiplexed user on subcarrier $n$, i.e., $\tilde{\eta}_{k}^{n}$ where $k \in \mathcal{U}_{n}$ [28, Section 6.2] and is defined as follows:

$$
\boldsymbol{\pi}_{n} \triangleq\left(\pi_{n}(1), \pi_{n}(2), \ldots, \pi_{n}\left(\left|\mathcal{U}_{n}\right|\right)\right),
$$

such that the following two criteria are satisfied:

1) The normalized noise power of multiplexed users on subcarrier $n$ are arranged in descending order:

$\tilde{\eta}_{\pi_{n}(1)}^{n} \geq \tilde{\eta}_{\pi_{n}(2)}^{n} \geq \cdots \geq \tilde{\eta}_{\pi_{n}\left(\left|\mathcal{U}_{n}\right|\right)}^{n}$

2) When there is a tie, we arrange those users in ascending order of their indices, i.e.,

if $\tilde{\eta}_{\pi_{n}(i)}^{n}=\tilde{\eta}_{\pi_{n}(j)}^{n}$ and $i<j$, then $\pi_{n}(i)<\pi_{n}(j)$.

We use Shannon capacity formula to model the capacity of a communication link. For $k \in \mathcal{K}$, and $n \in \mathcal{N}$, let $R_{k}^{n}$ be the achievable data rate of user $k$ on subcarrier $n$. Once the decoding order is determined according to the normalized receiver noise power, $R_{k}^{n}$ can be obtained as

$$
R_{k}^{n} \triangleq W_{n} \log _{2}\left(1+\frac{p_{k}^{n}}{\sum_{j=\pi_{n}^{-1}(k)+1}^{\left|\mathcal{U}_{n}\right|} p_{\pi_{n}(j)}^{n}+\tilde{\eta}_{k}^{n}}\right),
$$

where $\pi_{n}^{-1}(k)$ represents the decoding order of user $k$ in $\boldsymbol{\pi}_{n}$. More precisely, $\pi_{n}^{-1}(k)=i$ if $\pi_{n}(i)=k$.

The sum rate of the downlink MC-NOMA system is given by

$$
R_{\text {sum }} \triangleq \sum_{k=1}^{\mathcal{K}} \sum_{n=1}^{N} R_{k}^{n}
$$

\section{B. Problem Formulation}

The objective of this work is to maximize the sum of data rates subject to power constraints and a given maximum allowable number of multiplexed users per subcarrier. Mathematically, the problem can be formulated as follows:

$$
\text { maximize } R_{\text {sum }} \text {, }
$$

subject to

$$
\begin{array}{ll}
C 1: & \sum_{n=1}^{N} p_{k}^{n} \leq \bar{p}_{k}, k \in \mathcal{K}, \\
C 2: & p_{k}^{n} \geq 0, k \in \mathcal{K}, n \in \mathcal{N}, \\
C 3: & \left|\mathcal{U}_{n}\right| \leq M, \mathcal{U}_{n} \subseteq \mathcal{K}, n \in \mathcal{N}, \\
C 4: & p_{k}^{n}=0, k \notin \mathcal{U}_{n}, n \in \mathcal{N},
\end{array}
$$

where $C 1$ represents the maximum power allocation for user $k$. Such a constraint is imposed to guarantee the effort fairness among users as mentioned in Section II-A. $C 2$ indicates the non-negativity of the allocated power for each user on each subcarrier. $C 3$ restricts that the number of multiplexed users on each subcarrier is no more than $M$. When $M=1$, the system reduces to orthogonal multiple access (OMA). In NOMA, we consider $M \geq 2$. Note that $\mathcal{U}_{n}$ is a set variable to be optimized, which represents the subset of users to whom subcarrier $n$ is assigned. Besides, $C 4$ represents the power constraint of user $k$ on its unallocated subcarriers.

Note that the above maximization problem (3) is a coupled mixed integer non-convex problem and is known NP-hard [25], which is in general difficult to solve. For this reason, a closeto-upper-bound solution based on LDDP has been proposed in [25]. In the following, we will design another subcarrier and power allocation optimization method, which is more time efficient at the cost of slight degradation in sum rate performance.

\section{The Three-STEP OPTIMIZATION FraMeWORK FOR MC-NOMA}

The sum-rate performance of a scheme in MC-NOMA system is principally affected by two interacted factors, namely, subcarrier allocation and power control for multiplexed users. To solve the above MC-NOMA subcarrier allocation and power control problem efficiently, a three-step optimization methodology is proposed. We use the first two steps to complete subcarrier allocation. Specifically, Step 1 is applied to calculate the achievable data rate of each user on each subcarrier, which will be used to determine the final subcarrier assignment in Step 2 with the consideration of constraint $C 3$. Then, based on the subcarrier assignment obtained we allocate power for the multiplexed users in the third step.

For $k \in \mathcal{K}$, let $\mathcal{N}_{k} \subseteq \mathcal{N}$ be the set of subcarriers allocated to user $k$. Note that with a given subcarrier assignment, the 
above optimization problem (3) can be relaxed to the following power control sub-problem:

$$
\text { maximize } R_{\text {sum }}
$$

subject to

$$
\begin{aligned}
& C 1^{\prime}: \quad \sum_{n \in \mathcal{N}_{k}} p_{k}^{n} \leq \bar{p}_{k}, \quad k \in \mathcal{K}, \\
& C 2: \quad p_{k}^{n} \geq 0, \quad k \in \mathcal{K}, \quad n \in \mathcal{N}, \\
& C 3^{\prime}: \quad p_{k}^{n}=0, \quad k \in \mathcal{K}, \quad n \notin \mathcal{N}_{k} .
\end{aligned}
$$

We detail the three-step methodology below:

Step 1: To begin with, we relax constraints $C 3$ and $C 4$ such that all users can use all $N$ subcarriers simultaneously, i.e., $\mathcal{N}_{k}=\mathcal{N}$ for all $k \in \mathcal{K}$. We then solve the relaxed problem (5), which is a sub-problem of (3). We will prove in Section IV that this optimization problem is convex and can be optimally solved using a centralized gradient descent algorithm or some distributed power control methods. The detailed analysis about the centralized and distributed power control strategies are given in Section IV and Section V, respectively.

Step 2: We assign subcarriers to users based on the power allocation solution obtained in Step 1. For $n \in \mathcal{N}$,

- If $M$ or more users have positive power on subcarrier $n$, allocate subcarrier $n$ to the $M$ users who have the top- $M$ highest individual data rates on subcarrier $n$, with ties broken arbitrarily;

- If less than $M$ (but not equal to 0) users have positive power on subcarrier $n$, allocate subcarrier $n$ only to these users;

- If no one has positive power on subcarrier $n$, allocate subcarrier $n$ to user $k^{*}$, where $k^{*} \triangleq$ $\operatorname{argmin}_{k \in \mathcal{K}} \tilde{\eta}_{k}^{n}$, with ties broken arbitrarily.

After Step $2, \mathcal{U}_{n}$ is determined and satisfies $\left|\mathcal{U}_{n}\right| \leq M$ for all $n \in \mathcal{N}$.

Step 3: Using the subcarrier assignment obtained in Step 2, we revisit the optimization problem (5) to optimize and compute the final power allocation.

Note that in our case and problem, when Step 3 is conducted, the system performance cannot be further improved by adopting another Step 2. The reason is that after Step 3 is conducted, the set of users who are allowed to use subcarrier $n$, denoted by $\mathcal{U}_{n}$, has been determined for all $n$. It is a legitimate assignment in that $\left|\mathcal{U}_{n}\right| \leq M$ where $n \in \mathcal{N}$. Moreover, this subcarrier assignment will be different from that after Step 2 only if there are inactive subcarriers which were originally active after Step 1. We suspect that such a scenario will never occur, and even if it could occur, the probability would be extremely low. That is also why we have the three-step methodology.

\section{Centralized Power Allocation with Fixed SUBCARRIER ASSIGNMENT}

The power allocation problem (5) is central to the proposed three-step optimization scheme. We find that it is convex. The result is detailed below.
Let

$$
\boldsymbol{p}_{k} \triangleq\left(p_{k}^{n}\right)_{n \in \mathcal{N}_{k}} .
$$

Therefore, the set of all feasible powers for user $k$ can be given by

$$
\mathcal{P}_{k} \triangleq\left\{\boldsymbol{p}_{k}: \sum_{n \in \mathcal{N}_{k}} p_{k}^{n} \leq \bar{p}_{k} \text { and } p_{k}^{n} \geq 0, n \in \mathcal{N}_{k}\right\} .
$$

Define $\mathcal{P}$ as the set of feasible powers for the system, which can be expressed by the Cartesian product of all users' feasible sets and is given by

$$
\mathcal{P} \triangleq \prod_{k \in \mathcal{K}} \mathcal{P}_{k}
$$

Let $\boldsymbol{p} \triangleq\left(\boldsymbol{p}_{1}, \boldsymbol{p}_{2}, \ldots, \boldsymbol{p}_{K}\right)$. The convexity of problem (5) is proved below.

\section{Theorem 1. The maximization problem (5) is convex.}

Proof: The basic idea is to check that the feasible power region is non-empty and convex, and the objective function is concave. The feasible power region is non-empty because the zero vector satisfies constraints (6). Its convexity follows directly from (7), (8) and (9).

It remains to show that $R_{\text {sum }}$ is a concave function of $\boldsymbol{p}$. To prove this, we rewrite (2) as follows:

$$
\begin{aligned}
& R_{\text {sum }}=\sum_{n=1}^{N} W_{n} \sum_{k=1}^{K} \log _{2}\left(1+\frac{p_{k}^{n}}{\sum_{j=\pi_{n}^{-1}(k)+1}^{\left|\mathcal{U}_{n}\right|} p_{\pi_{n}(j)}^{n}+\tilde{\eta}_{k}^{n}}\right) \\
& =\sum_{n=1}^{N} W_{n} \sum_{i=1}^{\left|\mathcal{U}_{n}\right|} \log _{2}\left(1+\frac{p_{\pi_{n}(i)}^{n}}{\sum_{j=i+1}^{\left|\mathcal{U}_{n}\right|} p_{\pi_{n}(j)}^{n}+\tilde{\eta}_{\pi_{n}(i)}^{n}}\right) \\
& =\sum_{n=1}^{N} W_{n} \sum_{i=1}^{\left|\mathcal{U}_{n}\right|} \log _{2}\left(\frac{\sum_{j=i}^{\left|\mathcal{U}_{n}\right|} p_{\pi_{n}(j)}^{n}+\tilde{\eta}_{\pi_{n}(i)}^{n}}{\sum_{j=i+1}^{\left|\mathcal{U}_{n}\right|} p_{\pi_{n}(j)}^{n}+\tilde{\eta}_{\pi_{n}(i)}^{n}}\right) \\
& =\sum_{n=1}^{N} W_{n} \log _{2}\left(\frac{\sum_{j=1}^{\left|\mathcal{U}_{n}\right|} p_{\pi_{n}(j)}^{n}+\tilde{\eta}_{\pi_{n}(1)}^{n}}{\sum_{j=2}^{\left|\mathcal{U}_{n}\right|} p_{\pi_{n}(j)}^{n}+\tilde{\eta}_{\pi_{n}(1)}^{n}}\right. \\
& \left.\times \frac{\sum_{j=2}^{\left|\mathcal{U}_{n}\right|} p_{\pi_{n}(j)}^{n}+\tilde{\eta}_{\pi_{n}(2)}^{n}}{\sum_{j=3}^{\left|\mathcal{U}_{n}\right|} p_{\pi_{n}(j)}^{n}+\tilde{\eta}_{\pi_{n}(2)}^{n}} \times \cdots \times \frac{p_{\pi_{n}\left(\left|\mathcal{U}_{n}\right|\right)}^{n}+\tilde{\eta}_{\pi_{n}\left(\left|\mathcal{U}_{n}\right|\right)}^{n}}{\tilde{\eta}_{\pi_{n}\left(\left|\mathcal{U}_{n}\right|\right)}^{n}}\right) \\
& =\sum_{n=1}^{N} W_{n} \log _{2}\left(\frac{\sum_{j=1}^{\left|\mathcal{U}_{n}\right|} p_{\pi_{n}(j)}^{n}+\tilde{\eta}_{\pi_{n}(1)}^{n}}{\tilde{\eta}_{\pi_{n}\left(\left|\mathcal{U}_{n}\right|\right)}^{n}}\right. \\
& \left.\times \frac{\sum_{j=2}^{\left|\mathcal{U}_{n}\right|} p_{\pi_{n}(j)}^{n}+\tilde{\eta}_{\pi_{n}(2)}^{n}}{\sum_{j=2}^{\left|\mathcal{U}_{n}\right|} p_{\pi_{n}(j)}^{n}+\tilde{\eta}_{\pi_{n}(1)}^{n}} \times \cdots \times \frac{p_{\pi_{n}\left(\left|\mathcal{U}_{n}\right|\right)}^{n}+\tilde{\eta}_{\pi_{n}\left(\left|\mathcal{U}_{n}\right|\right)}^{n}}{p_{\pi_{n}\left(\left|\mathcal{U}_{n}\right|\right)}^{n}+\tilde{\eta}_{\pi_{n}\left(\left|\mathcal{U}_{n}\right|-1\right)}^{n}}\right) \\
& =\sum_{n=1}^{N} W_{n} \sum_{i=1}^{\left|\mathcal{U}_{n}\right|-1} \log _{2}\left(\frac{\sum_{j=i+1}^{\left|\mathcal{U}_{n}\right|} p_{\pi_{n}(j)}^{n}+\tilde{\eta}_{\pi_{n}(i+1)}^{n}}{\sum_{j=i+1}^{\left|\mathcal{U}_{n}\right|} p_{\pi_{n}(j)}^{n}+\tilde{\eta}_{\pi_{n}(i)}^{n}}\right) \\
& +\sum_{n=1}^{N} W_{n} \log _{2}\left(\frac{\sum_{j=1}^{\left|\mathcal{U}_{n}\right|} p_{\pi_{n}(j)}^{n}+\tilde{\eta}_{\pi_{n}(1)}^{n}}{\tilde{\eta}_{\pi_{n}\left(\left|\mathcal{U}_{n}\right|\right)}^{n}}\right) .
\end{aligned}
$$

For $n \in \mathcal{N}$ and $i \in \mathcal{U}_{n}$, let $\alpha_{i}^{n}(\boldsymbol{p}) \triangleq \frac{\sum_{j=i+1}^{\left|\mathcal{U}_{n}\right|} p_{\pi_{n}(j)}^{n}+\tilde{\eta}_{\pi_{n}(i+1)}^{n}}{\sum_{j=i+1}^{\left|\mathcal{U}_{n}\right|} p_{\pi_{n}(j)}^{n}+\tilde{\eta}_{\pi_{n}(i)}^{n}}$ 
and $\beta^{n}(\boldsymbol{p}) \triangleq \frac{\sum_{j=1}^{\left|\mathcal{U}_{n}\right|} p_{\pi_{n}(j)}^{n}+\tilde{\eta}_{\pi_{n}(1)}^{n}}{\tilde{\eta}_{\pi_{n}\left(\left|\mathcal{U}_{n}\right|\right)}^{n}}$, respectively. Thus, (10) can be re-written as

$$
R_{\text {sum }}=\sum_{n=1}^{N} W_{n}\left(\sum_{i=1}^{\left|\mathcal{U}_{n}\right|-1} \log _{2}\left(\alpha_{i}^{n}(\boldsymbol{p})\right)+\log _{2}\left(\beta^{n}(\boldsymbol{p})\right)\right) .
$$

It can be shown that $\alpha_{i}^{n}=1+\frac{\tilde{\eta}_{\pi_{n}(i+1)}^{n}-\tilde{\eta}_{\pi_{n}(i)}^{n}}{\sum_{j=i+1}^{\left|\mathcal{n}_{n}\right|} p_{\pi_{n}(j)}^{n}+\tilde{\eta}_{\pi_{n}(i)}^{n}}$ is a concave function of $\boldsymbol{p}$ [29] and $\beta^{n}$ is linear to $\boldsymbol{p}$. Hence, both $\alpha_{i}^{n}$ and $\beta^{n}$ are concave functions of $\boldsymbol{p}$. Moreover, $\alpha_{i}^{n} \geq 1$ as $\tilde{\eta}_{\pi_{n}(i+1)}^{n} \geq \tilde{\eta}_{\pi_{n}(i)}^{n}$. Thus, $\log _{2}\left(\alpha_{i}^{n}\right)$ and $\log _{2}\left(\beta^{n}\right)$ are concave functions because the logarithmic operation in our scenario is concave and non-decreasing. Since finite summation of concave functions is still concave [29], we deduce that $R_{\text {sum }}$ is concave. This completes the proof.

According to Theorem 1, the optimization problem (5) is convex. Therefore, it can be optimally solved through classical convex programming methods [30]. Since (5) is a linearly constrained convex problem, we choose the projected gradient descent method [31] to solve it. The pseudo-code of the projected gradient descent algorithm for solving (5) is given in Algorithm 1.

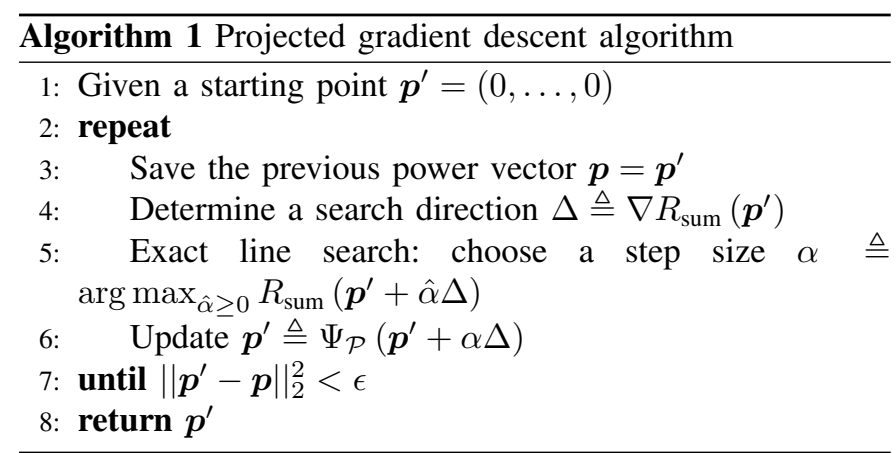

In Algorithm 1, the operator $\nabla$ represents the gradient with respect to power vector $\boldsymbol{p} . \Psi_{\mathcal{P}}: \mathbb{R}^{D} \rightarrow \mathcal{P}$ denotes the Euclidean projection on the feasible set $\mathcal{P}$, where $D \triangleq$ $\sum_{k=1}^{K}\left|\mathcal{N}_{k}\right|$ and $\mathbb{R}^{\Omega}$ is the set of $\Omega$-dimensional real vectors. $\epsilon$ is a small constant. Note that the projection $\Psi_{\mathcal{P}}(\boldsymbol{p})$ can be obtained by solving the following problem:

$$
\begin{array}{ll}
\text { minimize } & \|\boldsymbol{p}-\boldsymbol{x}\|_{2}^{2} \\
\text { subject to } & \boldsymbol{x} \in \mathcal{P},
\end{array}
$$

where $\|\cdot\|_{2}$ represents the $l_{2}$-norm of a vector. $\mathcal{P}$ is the Cartesian product of $\mathcal{P}_{k}$, which is given by (9).

Note that the detailed definitions of the projection $\Psi_{\mathcal{P}}$ and the convergence analysis of Algorithm 1 can be found in [32] and [31], respectively.

\section{Distributed Power Allocation with Fixed SUBCARRIER ASSIGNMENT}

In Section IV, we solve the maximization problem (5) through a standard convex programming method, which is however a centralized algorithm. In this section, we provide two distributed power allocation strategies by transforming the power control problem into a multi-player concave game. The distributed power control methods are more time efficient than the centralized scheme, which can balance the sum-rate performance and computational cost when considering practical applications. In addition, the techniques can be extended to solve similar problems. Specifically, one of the two distributed methods is based on pseudo-gradient algorithm and the other one on iterative waterfilling algorithm. The existence and uniqueness of Nash equilibrium of this game is demonstrated and pseudo-gradient algorithm can be applied to reach this point. In addition, the convergence of iterative waterfilling algorithm in some case is also proved. Besides the above results, we analyze the asymptotic computational complexity of the proposed power control strategies at the end of this section.

\section{A. Game-Theoretic Analysis}

In this subsection, the power control problem is first formulated in a game-theoretic manner. Subsequently, the existence and uniqueness of this game's Nash equilibrium are proved.

We consider distributed power allocation problem, in which each user allocates power to its assigned subcarriers so as to maximize its individual sum of data rates $R_{k} \triangleq \sum_{n \in \mathcal{N}_{k}} R_{k}^{n}$, $k \in \mathcal{K}$. Each user treats the interference from other users after SIC as additional white Gaussian noise (AWGN). From this point, the power control problem can be regarded as a $K$-player game [33], where $\boldsymbol{p}_{k}$ in (7) and $\mathcal{P}_{k}$ in (8) are the power allocation strategy and the strategy set of player $k$, respectively. Note that $\mathcal{P}$ represents the strategy space of all players in the system and is defined in (9). The strategy space is the set of all possible configurations in the game.

For $k \in \mathcal{K}$, let $\boldsymbol{p}_{-k} \triangleq\left(\boldsymbol{p}_{1}, \boldsymbol{p}_{2}, \ldots, \boldsymbol{p}_{k-1}, \boldsymbol{p}_{k+1}, \ldots, \boldsymbol{p}_{K}\right)$. We define

$$
u_{k}\left(\boldsymbol{p}_{k}, \boldsymbol{p}_{-k}\right) \triangleq \sum_{n \in \mathcal{N}_{k}} R_{k}^{n},
$$

as the utility function of user $k$, in which $R_{k}^{n}$ is defined in (1). Additionally, let

$$
\boldsymbol{u} \triangleq\left(u_{1}, u_{2}, \ldots, u_{K}\right) .
$$

Based on the aforementioned definitions, this game is characterized by the pair $(\mathcal{P}, \boldsymbol{u})$. A common equilibrium concept in game theory is the Nash equilibrium, which is defined as follows:

Definition 2. Given the link gain matrix and the normalized noise power of each user, a power allocation strategy $\tilde{\boldsymbol{p}} \triangleq$ $\left(\tilde{\boldsymbol{p}}_{1}, \tilde{\boldsymbol{p}}_{2}, \cdots, \tilde{\boldsymbol{p}}_{K}\right) \in \mathcal{P}$ is called a Nash equilibrium if the following holds for all $k \in \mathcal{K}$ and any $\boldsymbol{p}_{k} \in \mathcal{P}_{k}$,

$$
u_{k}\left(\tilde{\boldsymbol{p}}_{k}, \tilde{\boldsymbol{p}}_{-k}\right) \geq u_{k}\left(\boldsymbol{p}_{k}, \tilde{\boldsymbol{p}}_{-k}\right) .
$$

At a Nash equilibrium, given that the other players fix their power allocation, no player would further increase the data rate unilaterally, i.e, no user has incentive to change its power strategy at a Nash equilibrium. The absence of Nash equilibrium indicates that the distributed system is inherently unstable [34].

The existence of Nash equilibrium in our MC-NOMA system is a corollary of the fundamental theorem in game theory [33], [35]-[37]. According to the notation of this work, it can be represented as follows: 
Theorem 3. If for each $k \in \mathcal{K},(i) \mathcal{P}_{k}$ is compact and convex, (ii) $u_{k}$ is continuous in $\boldsymbol{p}$, and (iii) $u_{k}$ is concave in $\boldsymbol{p}_{k}$ for any given $\boldsymbol{p}_{-k}$, then $(\mathcal{P}, \boldsymbol{u})$ is called a concave game, and it has at least one Nash Equilibrium.

It is straightforward to see that all three conditions in the theorem are satisfied. Thus, there exists at least one Nash equilibrium in game $(\mathcal{P}, \boldsymbol{u})$.

Next, we show that the Nash equilibrium of $(\mathcal{P}, \boldsymbol{u})$ is unique. Let $\Phi: \mathcal{P} \rightarrow \mathbb{R}^{D}$ be the pseudo-gradient of $\boldsymbol{u}$ as defined in [33], i.e.,

$$
\Phi(\boldsymbol{p}) \triangleq\left(\nabla_{1} u_{1}(\boldsymbol{p}), \ldots, \nabla_{K} u_{K}(\boldsymbol{p})\right), \forall \boldsymbol{p} \in \mathcal{P},
$$

in which $\nabla_{k}$ denotes the gradient with respect to user $k$ 's power vector $\boldsymbol{p}_{k}$, i.e.,

$$
\nabla_{k} u_{k}(\boldsymbol{p})=\left(\frac{W_{n}}{\ln 2} \cdot \frac{1}{\sum_{j=\pi_{n}^{-1}(k)}^{\left|\mathcal{U}_{n}\right|} p_{\pi_{n}(j)}^{n}+\tilde{\eta}_{k}^{n}}\right)_{n \in \mathcal{N}_{k}} .
$$

Since the uniqueness of Nash equilibrium is related to the monotonicity of $\Phi$ [33], we will show that $\Phi$ is strongly monotone. Let $\mathbb{R}_{++}^{Q}$ be the set of $Q$-dimensional strictly positive real vectors. An auxiliary lemma is introduced as follows:

Lemma 4. Let $\left(b_{i}\right)_{i \in\{1 \cdots Q\}} \in \mathbb{R}_{++}^{Q}$ be a non-increasing sequence with $Q$ positive real values, i.e., $b_{1} \geq b_{2} \geq \cdots \geq$ $b_{Q-1} \geq b_{Q}>0$. Then, for all $\left(x_{i}\right)_{i \in\{1 \cdots Q\}} \in \mathbb{R}^{Q}$,

$$
\sum_{i=1}^{Q} \sum_{j=i}^{Q} \frac{x_{i} x_{j}}{b_{i}} \geq \frac{\sum_{i=1}^{Q} x_{i}^{2}}{2 b_{i}} \geq \frac{\sum_{i=1}^{Q} x_{i}^{2}}{2 b_{1}} .
$$

Proof: The second inequality in (18) is obvious. We will prove the first inequality by mathematical induction.

Basis: It is evident that the statement is true for $Q=1$.

Inductive step: Assume that it is true for $Q=q$, i.e.,

$$
\sum_{i=1}^{q} \sum_{j=i}^{q} \frac{x_{i} x_{j}}{b_{i}} \geq \frac{\sum_{i=1}^{q} x_{i}^{2}}{2 b_{i}}
$$

Consider the case where $Q=q+1$. According to (18), we have

$$
\begin{aligned}
& \sum_{i=1}^{q+1} \sum_{j=i}^{q+1} \frac{x_{i} x_{j}}{b_{i}}-\sum_{i=1}^{q+1} \frac{x_{i}^{2}}{2 b_{i}} \\
& =\frac{x_{q+1}^{2}}{b_{q+1}}+\frac{x_{q}^{2}}{b_{q}}+\frac{x_{q} x_{q+1}}{b_{q}}+\sum_{i=1}^{q-1} \sum_{j=i}^{q+1} \frac{x_{i} x_{j}}{b_{i}} \\
& -\left(\frac{x_{q+1}^{2}}{2 b_{q+1}}+\frac{x_{q}^{2}}{2 b_{q}}+\sum_{i=1}^{q-1} \frac{x_{i}^{2}}{2 b_{i}}\right) \\
& \geq \frac{x_{q+1}^{2}}{2 b_{q}}+\frac{x_{q}^{2}}{2 b_{q}}+\frac{x_{q} x_{q+1}}{b_{q}}+\sum_{i=1}^{q-1} \sum_{j=i}^{q+1} \frac{x_{i} x_{j}}{b_{i}}-\sum_{i=1}^{q-1} \frac{x_{i}^{2}}{2 b_{i}} \\
& =\frac{\left(x_{q+1}+x_{q}\right)^{2}}{2 b_{q}}+\sum_{i=1}^{q-1} \sum_{j=i}^{q+1} \frac{x_{i} x_{j}}{b_{i}}-\sum_{i=1}^{q-1} \frac{x_{i}^{2}}{2 b_{i}} .
\end{aligned}
$$

Note that the inequality in (20) holds because $b_{q+1} \leq b_{q}$.
We define an auxiliary variable $x_{i}^{\prime}$ as follows:

$$
x_{i}^{\prime} \triangleq\left\{\begin{array}{cl}
x_{i} & \text { if } i<q \\
x_{q+1}+x_{q} & \text { if } i=q .
\end{array}\right.
$$

Therefore, the last expression in (20) can be re-written as

$$
\begin{aligned}
& \frac{\left(x_{q+1}+x_{q}\right)^{2}}{2 b_{q}}+\sum_{i=1}^{q-1} \sum_{j=i}^{q+1} \frac{x_{i} x_{j}}{b_{i}}-\sum_{i=1}^{q-1} \frac{x_{i}^{2}}{2 b_{i}} \\
& =\frac{x_{q}^{\prime 2}}{2 b_{q}}+\left(\sum_{i=1}^{q} \sum_{j=i}^{q} \frac{x_{i}^{\prime} x_{j}^{\prime}}{b_{i}}-\frac{x_{q}^{\prime 2}}{b_{q}}\right)-\sum_{i=1}^{q-1} \frac{x_{i}^{\prime 2}}{2 b_{i}} \\
& =-\frac{x_{q}^{\prime 2}}{2 b_{q}}+\sum_{i=1}^{q} \sum_{j=i}^{q} \frac{x_{i}^{\prime} x_{j}^{\prime}}{b_{i}}-\sum_{i=1}^{q-1} \frac{x_{i}^{\prime 2}}{2 b_{i}} \\
& \geq-\frac{x_{q}^{\prime 2}}{2 b_{q}}+\frac{x_{q}^{\prime 2}}{2 b_{q}} \text { by induction hypothesis } \\
& =0 .
\end{aligned}
$$

Therefore, the statement is true for $Q=q+1$, which completes the proof.

With the aforementioned lemma, we have the following theorem:

Theorem 5. $\Phi$ is strongly monotone, i.e., there exists a constant $c<0$ such that for all $\boldsymbol{p}, \boldsymbol{p}^{\prime} \in \mathcal{P}$,

$$
\left(\Phi(\boldsymbol{p})-\Phi\left(\boldsymbol{p}^{\prime}\right)\right)^{T} \cdot\left(\boldsymbol{p}-\boldsymbol{p}^{\prime}\right) \leq c\left\|\boldsymbol{p}-\boldsymbol{p}^{\prime}\right\|_{2}^{2},
$$

in which $\Phi$ is defined in (16).

Proof: For all $\boldsymbol{p}, \boldsymbol{p}^{\prime} \in \mathcal{P}$, according to (16) and (17), we have

$$
\left(\Phi(\boldsymbol{p})-\Phi\left(\boldsymbol{p}^{\prime}\right)\right)^{T} \cdot\left(\boldsymbol{p}-\boldsymbol{p}^{\prime}\right)=\frac{W_{n}}{\ln 2} \sum_{k=1}^{K} \sum_{n=1}^{N}\left(\frac{1}{\hat{I}_{k}^{n}}-\frac{1}{\hat{I}_{k}^{\prime n}}\right)\left(p_{k}^{n}-p_{k}^{\prime n}\right),
$$

where

$$
\hat{I}_{k}^{n}=\sum_{j=\pi_{n}^{-1}(k)}^{\left|\mathcal{U}_{n}\right|} p_{\pi_{n}(j)}^{n}+\tilde{\eta}_{k}^{n},
$$

and

$$
\hat{I}_{k}^{\prime n}=\sum_{j=\pi_{n}^{-1}(k)}^{\left|\mathcal{U}_{n}\right|} p_{\pi_{n}(j)}^{\prime n}+\tilde{\eta}_{k}^{n} .
$$

We re-arrange (24) as follows:

$$
\begin{aligned}
& \left(\Phi(\boldsymbol{p})-\Phi\left(\boldsymbol{p}^{\prime}\right)\right)^{T} \cdot\left(\boldsymbol{p}-\boldsymbol{p}^{\prime}\right) \\
& =\frac{W_{n}}{\ln 2} \sum_{k=1}^{K} \sum_{n=1}^{N} \frac{\left(p_{k}^{n}-p_{k}^{\prime n}\right)\left(\sum_{j=\pi_{n}^{-1}(k)}^{\left|\mathcal{U}_{n}\right|}\left(p_{\pi_{n}(j)}^{\prime n}-p_{\pi_{n}(j)}^{n}\right)\right)}{\hat{I}_{k}^{n} \hat{I}_{k}^{\prime n}} \\
& =\frac{W_{n}}{\ln 2} \sum_{n=1}^{N} \sum_{i=1}^{\left|\mathcal{U}_{n}\right|} \frac{\left(p_{\pi_{n}(i)}^{n}-p_{\pi_{n}(i)}^{\prime n}\right)\left(\sum_{j=i}^{\left|\mathcal{U}_{n}\right|}\left(p_{\pi_{n}(j)}^{\prime n}-p_{\pi_{n}(j)}^{n}\right)\right)}{\hat{I}_{\pi_{n}(i)}^{n} \hat{I}_{\pi_{n}(i)}^{\prime n}} \\
& =-\frac{W_{n}}{\ln 2} \sum_{n=1}^{N} \sum_{i=1}^{\left|\mathcal{U}_{n}\right|} \sum_{j=i}^{\left|\mathcal{U}_{n}\right|} \frac{x_{i}^{n} x_{j}^{n}}{b_{i}^{n}},
\end{aligned}
$$


where $x_{i}^{n}=p_{\pi_{n}(i)}^{n}-p_{\pi_{n}(i)}^{\prime n}$ and

$$
\begin{aligned}
b_{i}^{n} & =\hat{I}_{\pi_{n}(i)}^{n} \hat{I}_{\pi_{n}(i)}^{\prime n} \\
& =\left(\sum_{j=i}^{\left|\mathcal{U}_{n}\right|} p_{\pi_{n}(j)}^{n}+\tilde{\eta}_{\pi_{n}(i)}^{n}\right)\left(\sum_{j=i}^{\left|\mathcal{U}_{n}\right|} p_{\pi_{n}(j)}^{\prime n}+\tilde{\eta}_{\pi_{n}(i)}^{n}\right),
\end{aligned}
$$

for $n \in \mathcal{N}$ and $i \in\left\{1,2, \ldots,\left|\mathcal{U}_{n}\right|\right\}$.

Note that

$$
\begin{aligned}
b_{1}^{n} & =\left(\sum_{j=1}^{\left|\mathcal{U}_{n}\right|} p_{\pi_{n}(j)}^{n}+\tilde{\eta}_{\pi_{n}(1)}^{n}\right)\left(\sum_{j=1}^{\left|\mathcal{U}_{n}\right|} p_{\pi_{n}(j)}^{\prime n}+\tilde{\eta}_{\pi_{n}(1)}^{n}\right) \\
& \leq\left(\sum_{j=1}^{\left|\mathcal{U}_{n}\right|} \bar{p}_{\pi_{n}(j)}+\tilde{\eta}_{\pi_{n}(1)}^{n}\right)^{2} \triangleq \frac{C_{n}}{2}
\end{aligned}
$$

Based on Lemma 4, we have

$$
\begin{aligned}
-\sum_{n=1}^{N} \frac{W_{n}}{\ln 2} \sum_{i=1}^{\left|\mathcal{U}_{n}\right|} \sum_{j=i}^{\left|\mathcal{U}_{n}\right|} \frac{x_{i}^{n} x_{j}^{n}}{b_{i}^{n}} & \leq-\sum_{n=1}^{N} \frac{W_{n}}{\ln 2 \cdot C_{n}} \sum_{i=1}^{\left|\mathcal{U}_{n}\right|}\left(x_{i}^{n}\right)^{2} \\
& \leq c\left\|\boldsymbol{p}-\boldsymbol{p}^{\prime}\right\|_{2}^{2},
\end{aligned}
$$

where $c$ is a constant such that $-\sum_{n=1}^{N} \frac{W_{n}}{\ln 2 \cdot C_{n}} \leq c<0$. This completes the proof.

Note that the detailed definition of strong monotonicity in Theorem 5 can be found in [38].

Theorem 6. The game $(\mathcal{P}, \boldsymbol{u})$ has unique Nash equilibrium.

Proof: According to Theorem 5, the pseudo-gradient of this game, $\Phi$, is strongly monotone. Based on [33], [38], the game has a unique Nash equilibrium.

\section{B. Two Distributed Power Control Algorithms}

In this subsection, we propose two distributed power allocation schemes, which can be applied in Step 1 and Step 3 of our three-step methodology to solve optimization problem (5).

1) The Pseudo-gradient Descent Method: The first scheme is based on pseudo-gradient descent method. It follows from Theorem 6 that the $K$-player game has a unique equilibrium point. Therefore, pseudo-gradient descent method can be applied to both Step 1 and Step 3 and it is guaranteed to converge to the unique fixed point [33].

For $k \in \mathcal{K}$ and $n \in \mathcal{N}_{k}$, we define

$$
\tilde{I}_{k}^{n} \triangleq \sum_{j=\pi_{n}^{-1}(k)+1}^{\left|\mathcal{U}_{n}\right|} p_{\pi_{n}(j)}^{n}+\tilde{\eta}_{k}^{n},
$$

as the normalized interference plus noise of user $k$ on subcarrier $n$.

Without loss of generality, we take user $k \in \mathcal{K}$ as an example and state the pseudo-code of the pseudo-gradient descent algorithm in Algorithm 2.

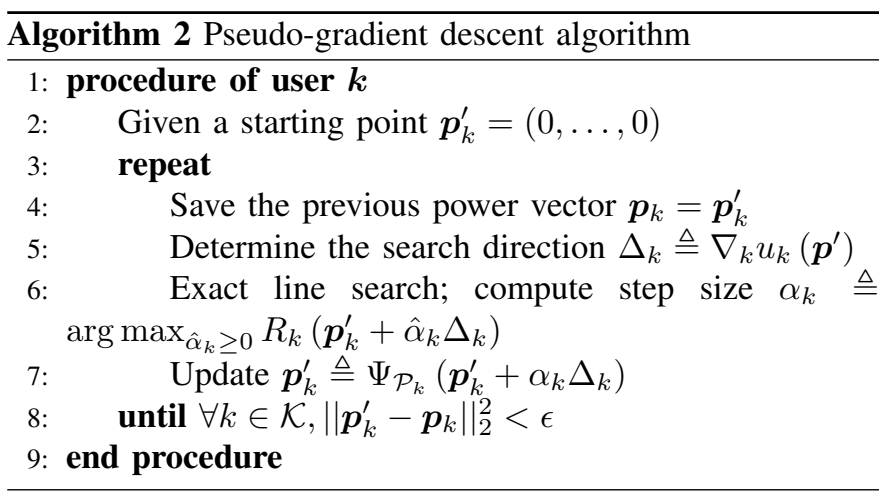

In Algorithm 2, $\Psi_{\mathcal{P}_{k}}$ represents the projection on $\mathcal{P}_{k}$, $k \in \mathcal{K}$. The pseudo-gradient descent algorithm is distributed in the sense that each user only needs to know the link gain between itself and the BS and the normalized interference plus noise on each subcarrier, i.e., $\tilde{I}_{k}^{n}$. This can be seen at Line 5 of Algorithm 2, in which $\nabla_{k} u_{k}$ is given by (17). All these information can be measured or estimated locally.

2) Iterative Waterfilling Method: The second scheme is based on iterative waterfilling algorithm (IWA). We show that its convergence can be guaranteed when $\left|\mathcal{U}_{n}\right| \leq 2$ for all $n \in \mathcal{N}$. To prove this, we first introduce the IWA and we focus on the synchronous version (SIWA), in which all players adjust their power allocation simultaneously.

Given fixed power allocation for other users and constant channel gains, i.e., $\tilde{I}_{k}^{n}$ are fixed, the optimal power allocation for user $k$ is given by the following theorem [39]:

Theorem 7. $\boldsymbol{p}_{k}^{*} \in \mathcal{P}_{k}$ maximizes $R_{k}$ if and only if there exists a water level, $\omega_{k}$, such that

$$
p_{k}^{* n}=\left[\omega_{k}-\tilde{I}_{k}^{n}\right]^{+}, \text {for } n \in \mathcal{N}_{k},
$$

where

$$
[X]^{+}=\left\{\begin{array}{cc}
0 & \text { if } X \leq 0 \\
X & \text { otherwise }
\end{array}\right.
$$

and

$$
\sum_{n \in \mathcal{N}_{k}} p_{k}^{* n}=\bar{p}_{k} .
$$

With the given power vectors of the other users, we define the waterfilling function for user $k$ as

$$
f_{k}\left(\boldsymbol{p}_{-k}\right) \triangleq\left(p_{k}^{* n}\right)_{n \in \mathcal{N}_{k},},
$$

where $p_{k}^{* n}$ is defined in Theorem 7. Furthermore, we define $F: \mathcal{P} \rightarrow \mathcal{P}$ as the waterfilling function of the system, which is given as follows:

$$
F\left(\boldsymbol{p}_{1}, \boldsymbol{p}_{2}, \ldots, \boldsymbol{p}_{K}\right) \triangleq\left(f_{k}\left(\boldsymbol{p}_{-k}\right)\right)_{k=1}^{K} .
$$

Note that SIWA is an iterative algorithm. For $k \in \mathcal{K}$ and $n \in \mathcal{N}_{k}$, let $p_{k}^{n}(t)$ be the power of user $k$ on subcarrier $n$ at time $t$, and $\boldsymbol{p}_{k}^{(t)}$ be the corresponding indexed family at time $t$. According to (26), we can define $\tilde{I}_{k}^{n}(t)$ as a function of $\left\{p_{j}^{n}(t): j \in \mathcal{U}_{n} \backslash\{k\}\right\}$. SIWA is then defined as follows:

$$
\left(\boldsymbol{p}_{1}^{(t+1)}, \boldsymbol{p}_{2}^{(t+1)}, \ldots, \boldsymbol{p}_{K}^{(t+1)}\right)=F\left(\boldsymbol{p}_{1}^{(t)}, \boldsymbol{p}_{2}^{(t)}, \ldots, \boldsymbol{p}_{K}^{(t)}\right),
$$

with $\boldsymbol{p}_{k}^{(0)}=0$ for all $k \in \mathcal{K}$. 
Let $\omega_{k}(t)$ be the water level of user $k$ at time $t$. From (29), we have

$$
\sum_{n \in \mathcal{N}_{k}}\left[\omega_{k}(t+1)-\tilde{I}_{k}^{n}(t)\right]^{+}=\bar{p}_{k} .
$$

Note that $\omega_{k}(t+1)$ can be regarded as a function of $\tilde{\boldsymbol{I}}_{k}(t) \triangleq$ $\left(\tilde{I}_{k}^{n}(t)\right)_{n \in N_{k}}$, and we denote it by

$$
\omega_{k}(t+1)=g_{k}\left(\tilde{\boldsymbol{I}}_{k}(t)\right) .
$$

Subsequently, we will investigate the convergence of SIWA. We consider two waterfilling scenarios for user $k$. The normalized interference at subcarrier $n$ in the two scenarios are denoted by $\tilde{I}_{k}^{n}$ and $\tilde{I}_{k}^{n^{\prime}}$, respectively. After performing waterfilling, we denote the water levels in the two scenarios by $\omega_{k}$ and $\omega_{k}^{\prime}$, respectively. With this setting, we have the following lemma:

Lemma 8. For any $k \in \mathcal{K}$, if $\tilde{I}_{k}^{n} \geq \tilde{I}_{k}^{n^{\prime}}$ for all $n \in \mathcal{N}_{k}$, then $g_{k}\left(\tilde{\boldsymbol{I}}_{k}\right) \geq g_{k}\left(\tilde{\boldsymbol{I}}_{k}^{\prime}\right)$.

Proof: Let $\omega_{k} \triangleq g_{k}\left(\tilde{\boldsymbol{I}}_{k}\right)$ and $\omega_{k}^{\prime} \triangleq g_{k}\left(\tilde{\boldsymbol{I}}_{k}^{\prime}\right)$. By contradiction, assume $\omega_{k}<\omega_{k}^{\prime}$. First, note that

$$
\sum_{n \in \mathcal{N}_{k}}\left[\omega_{k}-\tilde{I}_{k}^{n}\right]^{+} \leq \sum_{n \in \mathcal{N}_{k}}\left[\omega_{k}^{\prime}-\tilde{I}_{k}^{n}\right]^{+} \leq \sum_{n \in \mathcal{N}_{k}}\left[\omega_{k}^{\prime}-{\tilde{I_{k}^{n}}}^{\prime}\right]^{+},
$$

where the first inequality follows from the assumption that $\omega_{k}<\omega_{k}^{\prime}$ and the second inequality follows from the condition that $\tilde{I}_{k}^{n} \geq{\tilde{I_{k}^{n}}}^{\prime}$. According to (33), both sides are equal to $\bar{p}_{k}$, which implies, in particular, equality holds in the first inequality. This is possible only if

$$
\left[\omega_{k}-\tilde{I}_{k}^{n}\right]^{+}=\left[\omega_{k}^{\prime}-\tilde{I}_{k}^{n}\right]^{+}=0
$$

for all $n \in \mathcal{N}_{k}$. As a result, $\bar{p}_{k}=0$, which violates our assumption in the system model.

Theorem 9. Given $\left|\mathcal{U}_{n}\right| \leq 2$ for all $n \in \mathcal{N}$, SIWA always converges.

Proof: Since $\left|\mathcal{U}_{n}\right| \leq 2$, there are at most two multiplexed users in subcarrier $n$. For each subcarrier $n \in \mathcal{N}_{k}$, user $k$ may suffer from intra-band interference if subcarrier $n$ is also assigned to another user and that user has a smaller normalized noise power than user $k$. We denote this subset of subcarriers by $\mathcal{T}_{k}$, and its complement by $\mathcal{S}_{k}$, i.e., $\mathcal{S}_{k}=\mathcal{N}_{k} \backslash \mathcal{T}_{k}$. For $n \in \mathcal{T}_{k}$, we define $-k_{n}$ as the index of the user who shares subcarrier $n$ with user $k$.

Since each user has a total power constraint, $\tilde{I}_{k}^{n}(t)$ is bounded from above for all $k$ and $n$. Therefore, according to (33), $\omega_{k}(t)$ is also bounded from above for all $k \in \mathcal{K}$. The convergence of SIWA in Step 3 is established if

$$
\boldsymbol{\omega}(t) \triangleq\left(\omega_{1}(t), \omega_{2}(t), \ldots, \omega_{K}(t)\right)
$$

is monotonically increasing, i.e., for any $t \geq 1$,

$$
\boldsymbol{\omega}(t+1) \succeq \boldsymbol{\omega}(t),
$$

which we will prove in the following by induction.

Basis: Since $\boldsymbol{p}_{k}^{(0)}=0$ for all $k$, we have $\tilde{I}_{k}^{n}(0)=\tilde{\eta}_{k}^{n}$ for all $k$ and $n$. It is obvious that $\tilde{I}_{k}^{n}(1) \geq \tilde{I}_{k}^{n}(0)$. Lemma 8 and (34) imply $\boldsymbol{\omega}(2) \succeq \boldsymbol{\omega}(1)$.
Inductive step: Suppose (38) holds for $t=L$, i.e.,

$$
\boldsymbol{\omega}(L+1) \succeq \boldsymbol{\omega}(L) .
$$

First, consider $n \in \mathcal{S}_{k}$. By the definition of $\mathcal{S}_{k}, \tilde{I}_{k}^{n}(t)=\tilde{\eta}_{k}^{n}$ for all $t$, which implies

$$
\tilde{I}_{k}^{n}(L+1)=\tilde{I}_{k}^{n}(L), \quad \text { for } n \in \mathcal{S}_{k} .
$$

Next, consider $n \in \mathcal{T}_{k}$. According to (26), for any $t$, we have

$$
\tilde{I}_{k}^{n}(t)=p_{-k_{n}}^{n}(t)+\tilde{\eta}_{k}^{n}, \quad \text { for } n \in \mathcal{T}_{k} .
$$

By the definition of $\mathcal{T}_{k}$, user $-k_{n}$ experiences no intra-band interference in subcarrier $n$. The waterfilling method dictates that

$$
p_{-k_{n}}^{n}(t)=\left[\omega_{-k_{n}}(t)-\tilde{\eta}_{-k_{n}}^{n}\right]^{+} .
$$

Substituting it back to (41), we obtain

$$
\tilde{I}_{k}^{n}(t)=\left[\omega_{-k_{n}}(t)-\tilde{\eta}_{-k_{n}}^{n}\right]^{+}+\tilde{\eta}_{k}^{n}, \quad \text { for } n \in \mathcal{T}_{k},
$$

which, together with the inductive hypothesis in (39), implies

$$
\tilde{I}_{k}^{n}(L+1) \geq \tilde{I}_{k}^{n}(L), \quad \text { for } n \in \mathcal{T}_{k} .
$$

Invoking Lemma 8 with (40) and (44) and using (34), we obtain $\boldsymbol{\omega}(L+2) \succeq \boldsymbol{\omega}(L+1)$, which completes the proof. $\square$

Note that $M=2$ is commonly applied in practical NOMA system from implementation point of view [40]. According to the subcarrier assignment in Step 2 of the proposed threestep methodology, we have $\left|\mathcal{U}_{n}\right| \leq M$; therefore, if $M=2$, the convergence of SIWA in Step 3 is guaranteed based on Theorem 9.

\section{Asymptotic Computational Complexity Analysis}

In this subsection, we analyze the asymptotic computational complexity of the proposed centralized and distributed power allocation algorithms, which are the key components of our designed three-step resource allocation framework. Besides, the complexity analysis of both LDDP and FTPC is also discussed.

1) Complexity analysis of Algorithm 1: Since the optimization problem (5) is convex according to Theorem 1, it can be solved in a centralized manner by Algorithm 1 in $O(\log (1 / \epsilon))$ iterations [31], where $\epsilon$ is the error tolerance for algorithm termination. As shown in Line 4 of Algorithm 1, each iteration requires to compute the gradient $\nabla R_{\text {sum }}\left(\boldsymbol{p}^{\prime}\right)$ with respect to the power vector $\boldsymbol{p}^{\prime}$. Each element $\left(\nabla R_{\text {sum }}\right)_{k}^{n}$ of this gradient is computed by adding up the differentials of data rates $R_{\pi_{n}(j)}^{n}$, for $j<=\pi_{n}^{-1}(k)$, with respect to $p_{k}^{n}$. Therefore, each iteration has $O\left(N K^{2}\right)$ time complexity. In Step 3 of our three-step methodology, where no more than $M$ users are multiplexed on each subcarrier due to constraint $C 3$, this complexity can be further refined to $O\left(N M^{2}\right)$.

2) Complexity analysis of Algorithm 2: According to [33], Algorithm 2 is guaranteed to converge to the unique fixed point within $O(\log (1 / \epsilon))$ iterations. In Line 5 of Algorithm 2, each user $k \in \mathcal{K}$ only computes its pseudo-gradient $\nabla_{k} u_{k}\left(\boldsymbol{p}^{\prime}\right)$ which consists of $N$ elements. Thus, each iteration's time complexity is $O(N K)$. In Step 3 of our three-step method, this complexity can be further reduced to $O(N M)$. 
TABLE I

ASYMPTOTIC COMPUTATIONAL COMPLEXITY COMPARISON OF DIFFERENT SCHEMES

\begin{tabular}{|c|c|c|c|}
\hline Proposed Algorithms & Number of iterations & $\begin{array}{c}\text { Time complexity of } \\
\text { each iteration in Step 1 }\end{array}$ & $\begin{array}{c}\text { Time complexity of } \\
\text { each iteration in Step 3 }\end{array}$ \\
\hline Projected gradient descent (centralized) & $O(\log (1 / \epsilon))$ & $O\left(N K^{2}\right)$ & $O\left(N M^{2}\right)$ \\
\hline Pseudo-gradient descent (distributed) & $O(\log (1 / \epsilon))$ & $O(N K)$ & $O(N M)$ \\
\hline SIWA (distributed) & Exponentially fast (empirical) & $O(N K)$ & \multicolumn{3}{|c|}{ Time complexity } \\
\hline Benchmark Algorithms & \multicolumn{3}{|c|}{$O\left(C N M K J^{2}\right)$} \\
\hline LDDP & \multicolumn{3}{|c|}{$O(N K)$} \\
\hline FTPC & \multicolumn{3}{|l}{} \\
\hline
\end{tabular}

3) Complexity analysis of SIWA: By simulation results, we observe that SIWA converges exponentially fast. Since in Step 1 all users can use $N$ subcarriers simultaneously, each waterfilling step has $O(N K)$ time complexity. Furthermore, the time complexity comes to be $O(N M)$ in Step 3 as no more than $M$ users can be multiplexed on each subcarrier.

4) Complexity analysis of LDDP and FTPC: According to [25], the complexity of LDDP is $O\left(C N M K J^{2}\right)$, where $C$ represents the number of sub-gradient optimization iterations upon termination and $J$ is the number of power levels. In LDDP, the total power budget $P_{\text {total }}$ is divided in $J$ discrete power steps of value $P_{\text {total }} / J$ and power allocation is performed by distributing these discrete power steps among users and subcarriers. In section VI-D, we study the trade-off between sum-rate performance and computational complexity of LDDP for different values of $J$. We determine that for the system described in Table II, LDDP with $J=10 K$ is an appropriate choice as a near-optimal benchmark since its sumrate does not improve significantly by further increasing $J$. In addition, we analyze how the performance gain in LDDP's optimization depends on $J, N, K$ and $M$. We deduce that $J=O(\min \{K, M N\})$ is a good choice for achieving nearoptimal sum-rate in practice.

Besides, the complexity of FTPC is $O(N K)$ by definition. We summarize the above results in Table I.

\section{Vi. Simulation Results}

In this section, we evaluate the performance of the proposed subcarrier and power allocation strategies by extensive simulations. The radius $R$ of the cell is set to 250 meters. Within the cell, there is one BS located at the center and $K$ users uniformly distributed inside it. The system bandwidth $W$ is assumed to be $5 \mathrm{MHz}$ and $W_{n}=W / N$ for $n \in \mathcal{N}$, where $N=10$. The noise power spectral density is assumed to be $-174 \mathrm{dBm} / \mathrm{Hz}$. In the radio propagation model, we follow [41] and include the distance-dependent path loss, shadow fading and small-scale fading. The distance-dependent path loss is given by $128.1+37.6 \log _{10} d$, in which $d$ is the distance between the transmitter and the receiver in $\mathrm{km}$. Lognormal shadowing has a standard deviation of $8 \mathrm{~dB}$. For small-scale fading, each user experiences independent Rayleigh fading with variance 1 . The details of simulation parameters are summarized in Table II.

We will compare the performance of the following schemes:

- Double Iterative Waterfilling Algorithm (DIWA): we adopt the proposed three-step MC-NOMA optimization framework and use SIWA in Step 1 and 3;
- Double Gradient Algorithm (DGA): we adopt the proposed three-step MC-NOMA optimization framework and use the projected gradient descent algorithm (Algorithm 1) in both Step 1 and 3;

- Double Pseudo-Gradient Algorithm (DPGA): we apply the designed three-step MC-NOMA optimization scheme and adopt pseudo-gradient descent algorithm (Algorithm 2) in the Step 1 and Step 3;

- LDDP: the near-optimal scheme proposed in [25];

- NOMA-SIWA-FTPC: we apply the proposed SIWA to NOMA with FTPC, in which SIWA and FTPC are invoked to perform subcarrier and power allocation, respectively;

- OMA-FTPC: we adopt FTPC [2], [24], [42], [43] in orthogonal multiple access (OMA) system.

\section{A. The Convergence Time}

To begin with, we investigate the convergence time of the proposed power control algorithms. Fig. 1 shows the convergence of SIWA, DGA and DPGA in both Step 1 and Step 3 of our three-step resource allocation methodology. The number of users $K$ and the number of multiplexed users $M$ is set to be 10 and 2, respectively.

Fig. 1(a) and Fig. 1(b) show the convergence performance of SIWA in Step 1 and Step 3, respectively. We choose several users on an arbitrary chosen instance and use their water levels during iterations to demonstrate. The $x$-axis represents the number of iterations, while the $y$-axis denotes the water level of each user. We can observe that SIWA in both Step 1 and Step 3 takes only a few iterations to converge. As expected, the water level of each user in Step 3 is monotonically increasing. Fig. 1(c) illustrates the convergence of DGA (projected gradient descent algorithm) and DPGA (pseudo-gradient descent algorithm) in Step 1 and Step 3, respectively. The $x$-axis indicates the number of iterations, while the $y$-axis represents the sum of user data rates. It can be seen that these gradient descent based power control algorithms converge rapidly in both Steps 1 and 3.

\section{B. Sum of Data Rates}

In this subsection, we evaluate the sum of data rates performance of the aforementioned subcarrier and power allocation schemes. Note that each data point is obtained by averaging over 2,000 random instances.

The purpose of Fig. 2 and Fig. 3 is to compare the performance between NOMA system given by LDDP, our 


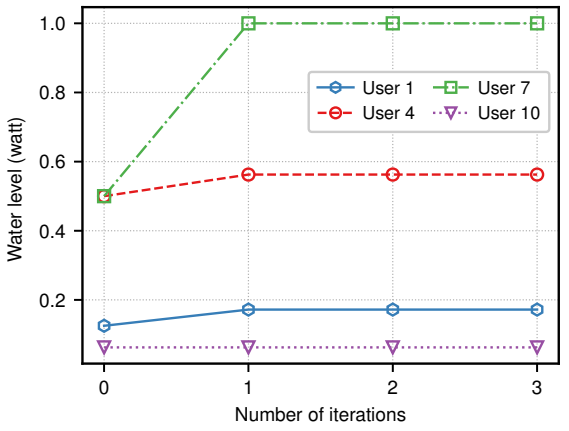

(a) Convergence of SIWA in Step 1

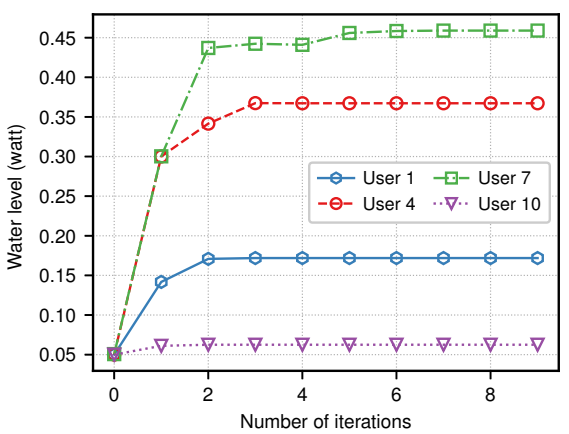

(b) Convergence of SIWA in Step 3

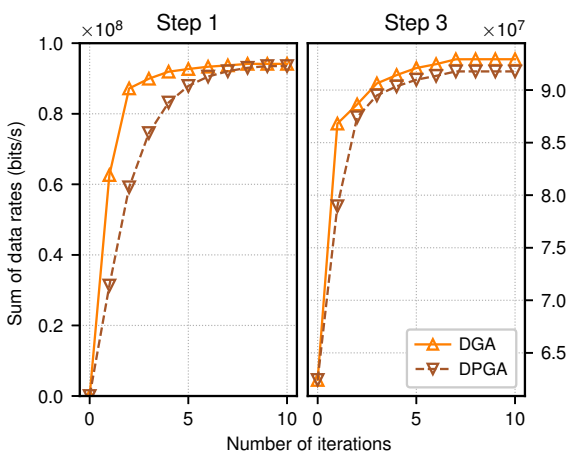

(c) Convergence of DGA and DPGA

Fig. 1. Convergence of the proposed power control algorithms.

TABLE II

SIMULATION PARAMETERS

\begin{tabular}{|c|c|}
\hline Parameters & Value \\
\hline Cell radius & $250 \mathrm{~m}$ \\
\hline Minimum distance from user to BS & $35 \mathrm{~m}$ \\
\hline Path loss & $128.1+37.6 \log _{10} d \mathrm{~dB}, d$ is in $\mathrm{km}$ \\
\hline Shadowing & Log-normal, standard deviation $8 \mathrm{~dB}$ \\
\hline Fading & Rayleigh fading with variance 1 \\
\hline Users distribution scheme & Randomly uniform distribution \\
\hline Noise power spectral density & $-174 \mathrm{dBm} / \mathrm{Hz}$ \\
\hline Overall system bandwidth, $W$ & $5 \mathrm{MHz}$ \\
\hline Subcarrier number, $N$ & 10 \\
\hline Number of users, $K$ & 3 to 20 \\
\hline Throughput calculation & 0.4 \\
\hline Decay factor of FTPC & Shannon's capacity formula \\
\hline Number of power levels of LDDP per user & 10$, ([0 W, $0.5 \mathrm{~W}]$, step by $0.05 \mathrm{~W})$ \\
\hline Power constraint for each user & $0.5 \mathrm{~W}$ \\
\hline Parameter $\epsilon$ & $10^{-4}$ \\
\hline Parameter $M$ & 1 (OMA), 2 to 6 (NOMA) \\
\hline
\end{tabular}

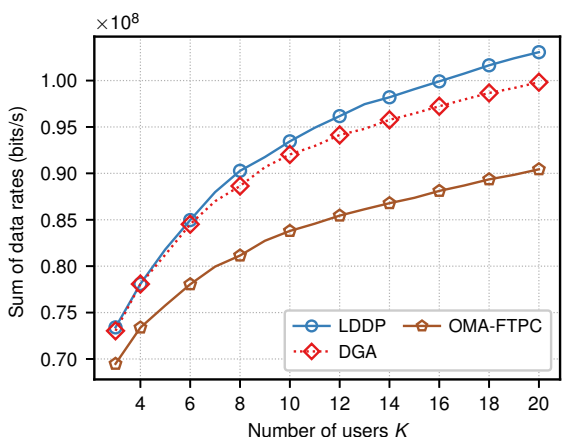

Fig. 2. Sum of data rates vs. different number of users, $M=2$

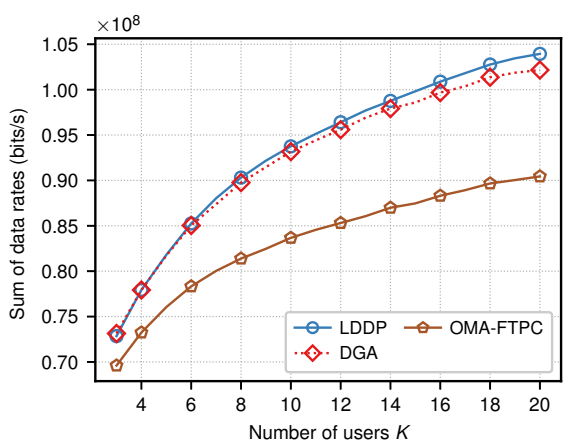

Fig. 3. Sum of data rates vs. different number of users, $M=3$

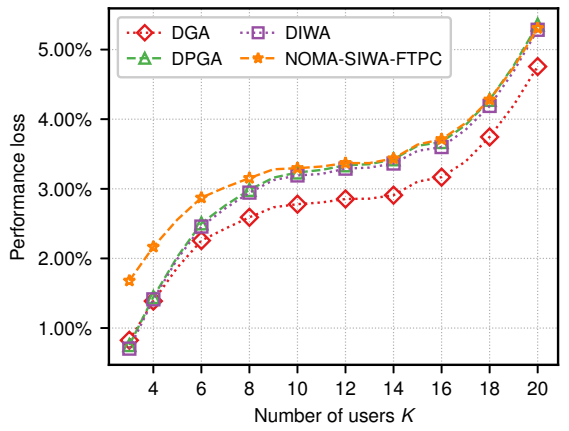

Fig. 4. Performance loss vs. different number of users, $M=2$

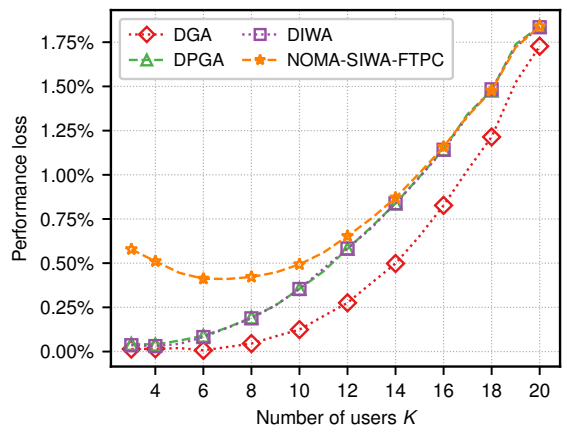

Fig. 5. Performance loss vs. different number of users, $M=3$ 


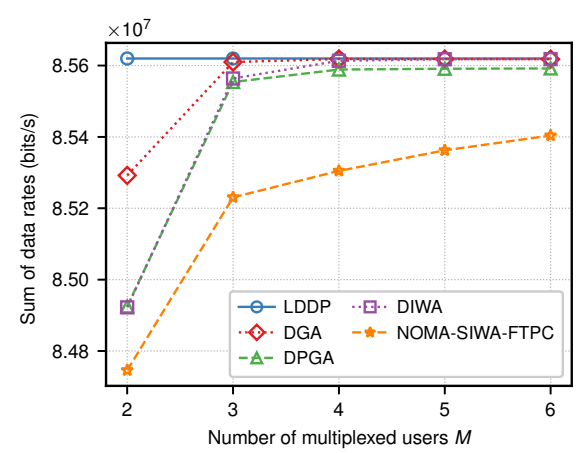

Fig. 6. Sum of data rates vs. different number of multiplexed users

three-step method and the performance in OMA system given by OMA-FTPC. Since projected gradient descent algorithm (Algorithm 1) achieves the optimal solution to power control problem (5), in Fig. 2 and Fig. 3, we choose to compare its sum-rate performance applied in the three-step algorithm (i.e., DGA according to our aforementioned definition) to that of LDDP and OMA-FTPC with different number of users. The number of multiplexed users $M$ of Fig. 2 and Fig. 3 is set to 2 and 3, respectively. This setting is based on an implementation point of view, i.e., reducing the receiver complexity and error propagation due to SIC [40].

In Fig. 2, it can be seen that the sum of data rates of each scheme increases with the number of users $K$. For any given $K$, LDDP has the best performance among the three schemes. Nevertheless, DGA achieves comparable sum of data rates to that of LDDP, see for example, when $K=20$ and $M=2$, the proposed DGA has a performance loss of only $3.14 \%$ when compared with LDDP. In addition, OMA-FTPC has the worst system performance among all. Similar conclusions can be drawn from Fig. 3. It is worth mentioning that the proposed scheme becomes more efficient with the increasing of $M$. For example, when $K=20$ and $M=3$, the performance loss of DGA when compared to LDDP is reduced to $1.71 \%$.

In Fig. 4 and Fig. 5, we compare the sum of data rates performance of the three-step scheme under DGA, DPGA, DIWA, and NOMA-SIWA-FTPC. The $x$-axis indicates the number of users, while the $y$-axis represents the sum-rate performance loss compared to that of LDDP, which is defined as follows:

$$
\frac{R_{\text {sum }}(L L D P)-R_{\text {sum }}}{R_{\text {sum }}(L L D P)},
$$

where $R_{\text {sum }}(L L D P)$ and $R_{\text {sum }}$ refer to the solutions of LDDP and the three-step algorithm, respectively.

Fig. 4 shows the performance loss versus different number of users $K$ of the four proposed schemes, where $M=2$. It can be seen that, for fixed $K$, DGA has the smallest performance loss among these four schemes. Meanwhile, DIWA has a similar performance to that of DPGA. In addition, for any given $K$, NOMA-SIWA-FTPC has the largest performance loss among all.

Fig. 5 represents the performance loss of our proposed resource allocation strategies when compared with LDDP where the number of multiplexed users is assumed to be 3 . The similar conclusions can be achieved to the scenario where 2 users are multiplexed on each subcarrier (Fig. 4), which are

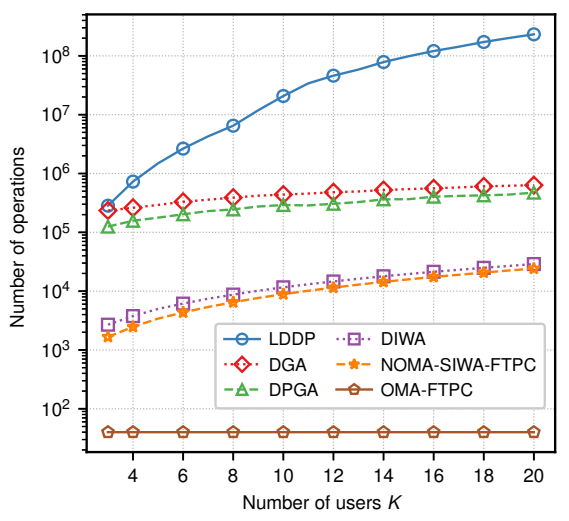

Fig. 7. Number of operations vs. different number of users, $M=2$

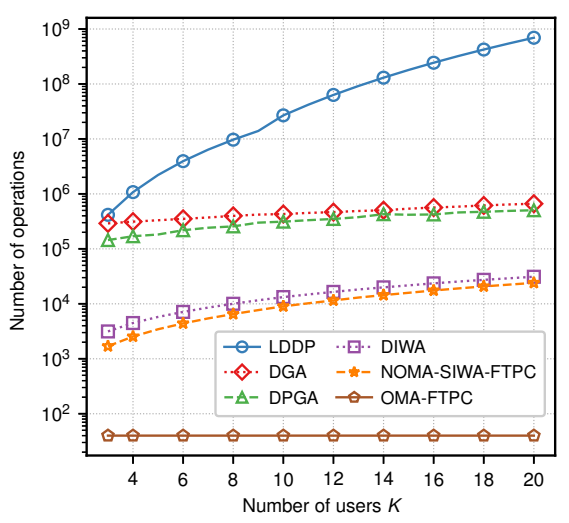

Fig. 8. Number of operations vs. different number of users, $M=3$

omitted here.

Fig. 6 depicts the sum of data rates of LDDP and our proposed resource allocation algorithms versus the number of multiplexed users $M$, in which the number of users $K$ is assumed to be 6. It can be seen that with the increasing of $M$, the performance gap between LDDP and our proposed schemes decreases. For example, when $M=4$, the proposed DGA and SIWA have almost the same performance to that of LDDP, i.e., a performance loss of approximately $0.0016 \%$ and $0.0074 \%$, respectively, while DPGA stagnates at $0.036 \%$ and NOMA-SIWA-FTPC has a performance loss of $0.37 \%$.

\section{Number of Operations}

Fig. 7 to Fig. 9 show the number of operations required by the different schemes. For each scheme, we count the number of additions, multiplications, and comparisons used, which reflects the time complexity, as an estimation.

In Fig. 7 and Fig. 8, the number of multiplexed users $M$ is fixed to 2 and 3 , respectively. We see that except OMAFTPC, the number of operations for each scheme increases with the increasing number of users, $K$. For any given $K$, DGA has the highest computational complexity among the proposed algorithms (DGA, DPGA, DIWA, NOMA-SIWAFTPC). OMA-FTPC requires the fewest operations of all. In addition, the proposed DIWA uses approximately the same number of operations as NOMA-SIWA-FTPC and slightly more operations than OMA-FTPC but much less operations 


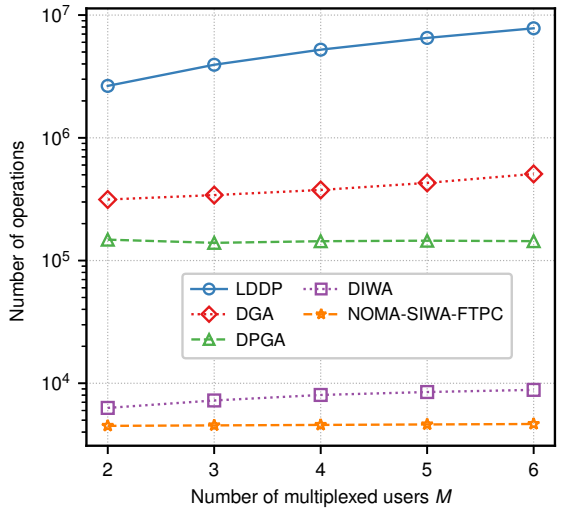

Fig. 9. Number of operations vs. different number of multiplexed users

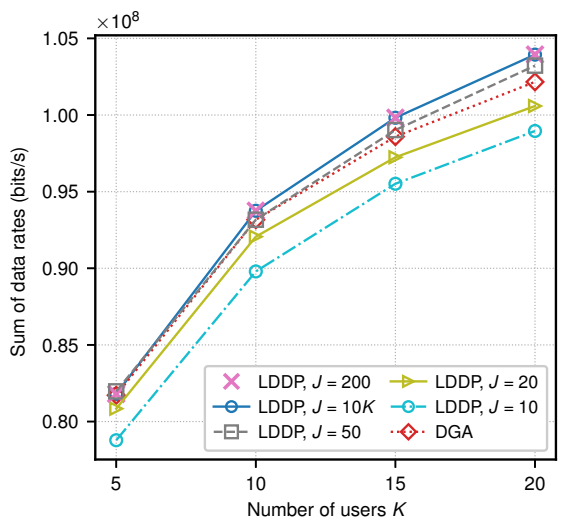

Fig. 10. Sum of data rates for different values of $J, M=3$

than DPGA and DGA. In any case, all of the proposed schemes are much more time efficient than LDDP especially when the number of users is large. For example, when $K=20$ and $M=2$, the number of operations required by DIWA, DPGA and DGA is less than $0.1 \%, 0.2 \%$, and $0.27 \%$ of that required by LDDP, respectively. Besides, when $K=20$ and $M=3$, the needed operations of DIWA, DPGA and DGA is around $0.01 \%, 0.07 \%$, and $0.1 \%$ of that needed by LDDP, respectively.

Fig. 9 compares the number of operations required under different number of multiplexed users $M$ with fixed number of users $K=6$. We can see that for any given $M$, the number of operations of LDDP is the highest of all. In addition, with the increasing of $M$, the computational complexity of LDDP has the fastest growth rate among all the schemes.

\section{Impact of the Power Levels $J$ on LDDP's Performance}

We show in this subsection how the sum-rate and complexity of LDDP depends on the number of power levels $J$ and the system's parameters. We present in Fig. 10 and Fig. 11 the sum-rate and number of operations of LDDP under different number of power levels $J=10,20,50,200$ and $10 K$, and compare them to the performance of DGA. As expected from [25], when $J$ increases, the sum-rate of LDDP increases until reaching the optimal, while the computational complexity increases in $O\left(C N M K J^{2}\right)$. The number of power levels considered here is always greater than 10 , otherwise

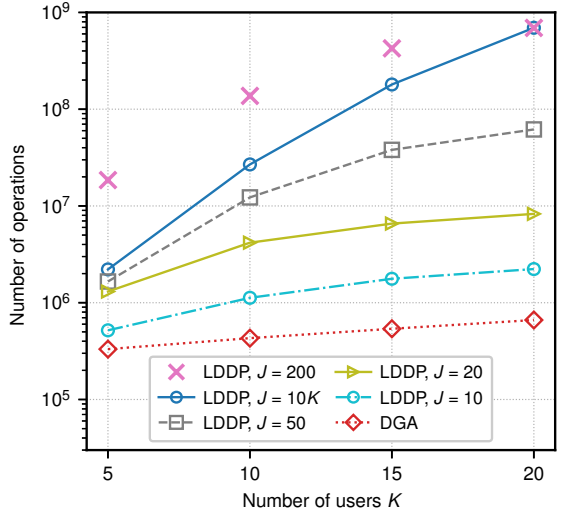

Fig. 11. Number of operations for different values of $J, M=3$

LDDP would not have enough discrete power steps to serve all $N=10$ subcarriers.

We observe in Fig. 10 that LDDP's sum-rate for $J=200$ and $J=10 \mathrm{~K}$ are very close, i.e. they have less than $0.01 \%$ of difference for any number of users. Hence, LDDP's sum-rate does not improve significantly by further increasing $J$ over $10 K$. Furthermore, the sum-rate is reduced significantly as $J$ decreases. This justifies the choice of LDDP with 10 power steps per user, i.e. $J=10 \mathrm{~K}$, as a benchmark near-optimal scheme in our simulations. Note that this value only holds for the system's parameters described in Table II. We discuss below how $J$ can be set in other systems depending on $N, K$ and $M$.

As the number of resource increases (subcarriers $N$, users $K, M$, etc.), $J$ should also increase in order to achieve similar gain in the joint subcarrier and power allocation. The idea is that each allocated user should have enough power steps to optimize the allocation of their individual power budget $\bar{p}_{k}$ among the subcarriers in LDDP. Given that the number of allocated user is at most $\min \{K, M N\}$, we choose empirically $J=O(\min \{K, M N\})$, which achieves good performance by simulation. For example, we choose $J=10 K$ in Fig. 10, since $K<M N=30$.

In Fig. 11, all LDDP schemes have higher complexity than DGA. Among these schemes, only LDDP with $J=$ $50,200,10 K$ achieve better sum-rate performance than DGA. The performance gains compared to DGA, for $K=20$, are respectively $1.04 \%$ and $1.74 \%$ for $J=50$ and $J=10 K=200$, while the increase in complexity are approximately 100 and 1000 folds. Moreover, DGA outperforms both LDDP with $J=10$ and $J=20$ in terms of higher sum-rate and lower complexity. For $K=20$, the performance loss of LDDP with $J=10$ and $J=20$ compared to DGA are respectively $3.20 \%$ and $1.54 \%$.

In summary, DGA has close to the best LDDP's sumrate, while requiring less computational complexity. The latter statement can be explained by comparing the asymptotic complexity of LDDP iteration $O\left(N M K J^{2}\right)$ and DGA iteration $O\left(N K^{2}\right)$. As discussed previously, we can assume $J=O(\min \{K, M N\})$. If $K \leq M N$ (which is the case considered in our simulations), LDDP iteration's complexity can be written as $O\left(N M K^{3}\right)$. In this case, DGA requires $M K$ times less operations than LDDP, which explains the results 


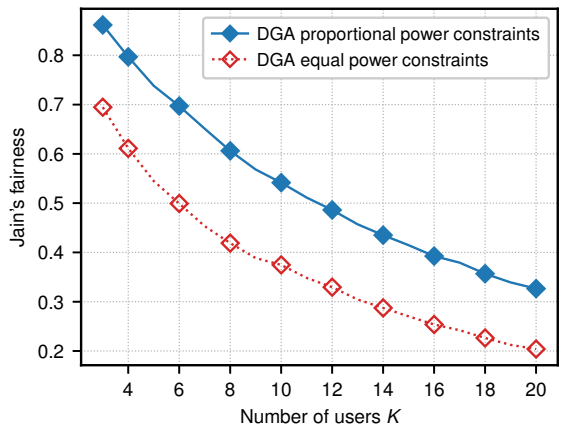

Fig. 12. Fairness index vs. different number of users, $M=2$

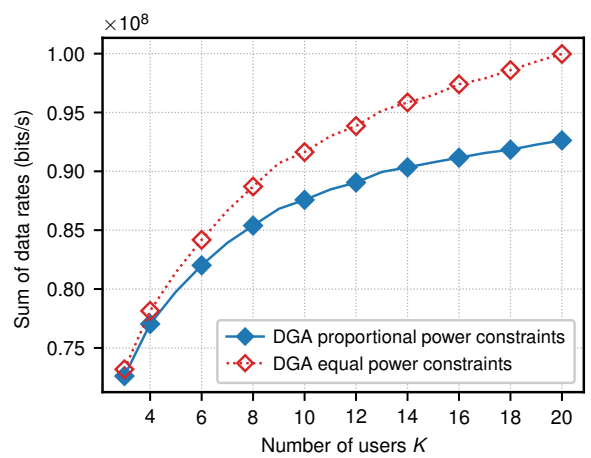

Fig. 13. Sum of data rates vs. different number of users, $M=2$

shown in Fig. 7, 8, 9 and Fig. 11. Otherwise if $K>M N$, then LDDP iteration's complexity becomes $O\left(N^{3} M^{3} K\right)$. This complexity is in practice higher than DGA's iteration complexity. Indeed, let's consider a 3GPP-LTE use case [44] with a bandwidth of $10 \mathrm{MHz}$ divided in $N=50$ resource blocks, and let $M=2$, DGA remains advantageous in terms of computational complexity as long as $K<N^{2} M^{3}$, i.e. up to 20,000 connected users.

\section{E. Fairness Performance}

As stated in Section II, the fairness among users can be obtained via adjusting the power constraint of individual user. We adopt Jain's fairness index [45] to show this performance, which is defined as $\frac{\left(\sum_{k=1}^{K} R_{k}\right)^{2}}{K \sum_{k=1}^{K} R_{k}^{2}}$. Fig. 12 depicts the fairness performance of the proposed scheme ${ }^{1}$ under different user power constraint strategies where the number of multiplexed user on each subcarrier is set to 2. Specifically, under DGA equal power constraints, each user has the same maximum power budget, i.e., $\bar{p}_{k}=P_{\text {total }} / K$, where $k \in \mathcal{K}$. Meanwhile, in DGA proportional power constraints, the power budget of user $k, \bar{p}_{k}$, is given as follows:

$$
\bar{p}_{k}=\frac{\left(\sum_{n \in \mathcal{N}} g_{k}^{n}\right)^{-1}}{\sum_{j \in \mathcal{K}}\left(\sum_{n \in \mathcal{N}} g_{j}^{n}\right)^{-1}} P_{\text {total }}, k \in \mathcal{K} .
$$

From Fig. 12 we see that, given the number of users, DGA under proportional power constraints has higher fairness index than that of DGA with equal power constraint. For example,

\footnotetext{
${ }^{1}$ For expression simplicity, we choose DGA as an example to demonstrate the fairness performance. Similar conclusions can be obtained for the other proposed resource allocation schemes.
}

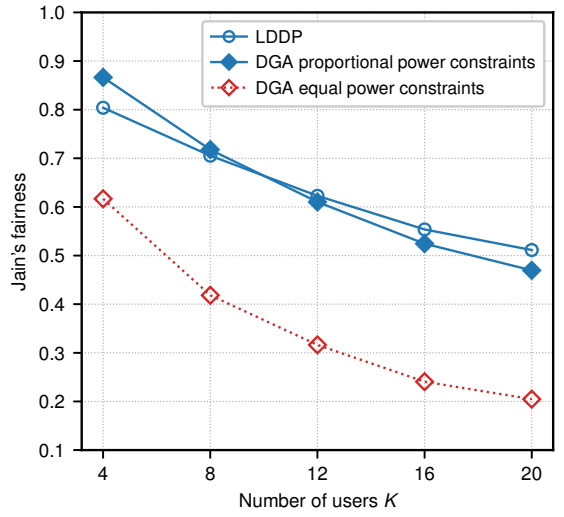

Fig. 14. Fairness index over a scheduling frame, $M=2$

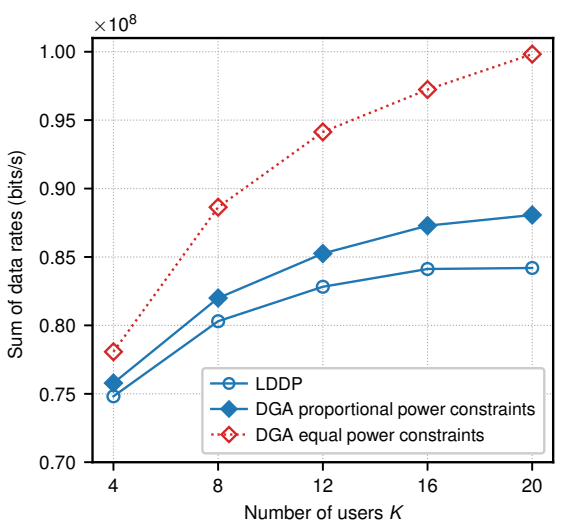

Fig. 15. Sum of data rates over a scheduling frame, $M=2$

when $K=20$, DGA with proportional power constraints improves Jain's fairness index by $60 \%$ when compared to that of equal power constraint scheme.

Fig. 13 is applied to illustrate the sum rate performance of DGA under different power constraint methods where two users are multiplexed on each subcarrier. For each value of $K$, DGA under equal power budget has better sum rate performance than DGA with proportional power constraints since the latter scheme assigns more power to users with weak channel conditions, resulting in sum rate performance degradation, e.g., when $K=20$, DGA with proportional power budget has $7.3 \%$ sum rate loss when compared to that of equal power budget.

\section{F. Fairness Performance over a Scheduling Frame}

In this subsection, we compare the fairness performance of DGA with proportional power constraint to LDDP with weighting factor proposed in [25]. In the same way as in [25], we consider a scheduling frame of $T=20$ times slots. The channel state information is collected at the beginning of the frame. Let $R_{k}(t)$ be user $k$ 's achievable data rate at time slot $t \in\{1, \ldots, T\}$. We define user $k$ 's average rate prior to time slot $t$ as follows:

$$
\bar{R}_{k}(t)=\left(1-\frac{1}{T}\right) \bar{R}_{k}(t-1)+\frac{1}{T} R_{k}(t) .
$$

At each time $t$, LDDP is applied to optimize the weighted sumrate $\sum_{k} w_{k}(t) R_{k}(t)$, where the weight $w_{k}(t)$ of each user $k$ 
is set inversely proportional to its average rate, i.e. $w_{k}(t)=$ $1 / \bar{R}_{k}(t)$. Besides, at each time $t$, the individual power budgets of DGA proportional power constraints are defined similarly to (46) with additional weight factors quoted below:

$$
\bar{p}_{k}(t)=\frac{w_{k}(t)\left(\sum_{n \in \mathcal{N}} g_{k}^{n}\right)^{-1}}{\sum_{j \in \mathcal{K}} w_{k}(t)\left(\sum_{n \in \mathcal{N}} g_{j}^{n}\right)^{-1}} P_{\text {total }}, k \in \mathcal{K} .
$$

Fig. 14 and Fig. 15 present respectively Jain's fairness index and sum-rate of the aforementioned schemes over a scheduling frame, in which DGA equal power constraints is also considered. Fig. 14 shows that LDDP and DGA proportional power constraints outperforms DGA equal power constraints in terms of fairness. This result is consistent with the fact that the latter scheme do not take into account previous data rates.

In Fig. 14, DGA proportional power constraints has greater fairness than LDDP for $K<10$, and inversely for $K \geq 10$. For $K=20$, LDDP's fairness index is $8.9 \%$ higher than that of DGA proportional power constraints. Nevertheless, we can see in Fig. 15 that DGA proportional power constraints achieves higher sum-rate than LDDP, with a performance gain of $4.6 \%$ for $K=20$.

In summary, the results of this section reveal the tradeoff between sum rate performance and computational cost, which can be balanced by using different resource allocation algorithms. Additionally, through adjusting the total power constraint of each user properly, a balancing between sum rate performance and user fairness can be obtained.

\section{CONCLUSION}

In this work, we investigate the sum rate maximization problem for the downlink of a MC-NOMA system. A threestep resource allocation methodology is proposed. First, we assume each of the users can use all subcarriers simultaneously and we solve the power control problem to get a power vector for each user; Second, we allocate subcarriers to users in a heuristic greedy manner with the obtained power vectors in Step 1; Third, the power allocation schemes used in Step 1 are applied once again to further enhance the system performance with the obtained subcarrier assignment of Step 2.

To solve the power control problem with fixed subcarrier assignment in both Step 1 and Step 3, a centralized method based on projected gradient descent algorithm is proposed. Besides, by transforming the power control problem into a multi-player concave game, two distributed power control strategies are proposed, which are more time efficient than the centralized scheme. Specifically, one of them is based on pseudo-gradient algorithm and the other one on iterative waterfilling algorithm. The existence and uniqueness of Nash equilibrium of this game is proved and pseudo-gradient algorithm can be applied to reach this point. In addition, the convergence of iterative waterfilling algorithm in some cases is also proved.

Simulation results show that the performance of the proposed three-step algorithms is comparable to LDDP but with much fewer computations. Among our proposed algorithms, DGA has the best sum-rate performance, while NOMA-SIWAFTPC is the most computationally efficient. The other two proposed algorithms, DPGA and DIWA, are able to strike a proper balance between sum rate and computation steps. In particular, DPGA has provable convergence guarantee while DIWA is more efficient in computation. Numerical results also demonstrate that through adjusting the total power budget of each user properly, a tradeoff between sum-rate performance and fairness performance can be achieved. Future work includes solving the joint subcarrier and power allocation for multi-cell MC-NOMA systems and other scenarios.

\section{REFERENCES}

[1] Y. Fu, L. Salaün, C. W. Sung, C. S. Chen, and M. Coupechoux, "Double iterative waterfilling for sum rate maximization in multicarrier NOMA systems," IEEE International Conference on Communications (ICC), pp. 1-6, May. 2017.

[2] Y. Saito, Y. Kishiyama, A. Benjebbour, T. Nakamura, A. Li, and K. Higuchi, "Non-orthogonal multiple access (NOMA) for cellular future radio access," IEEE Vehicular Technology Conference (VTC), pp. 1-5, Jun. 2013

[3] L. Dai, B. Wang, Y. Yuan, S. Han, C.-L. I, and Z. Wang, "Nonorthogonal multiple access for $5 \mathrm{G}$ : solutions, challenges, opportunities, and future research trends," IEEE Communications Magazine, vol. 53, no. 9, pp. 74-81, Sep. 2015.

[4] Z. Ding, P. Fan, and H. V. Poor, "Impact of user pairing on $5 \mathrm{G}$ nonorthogonal multiple access," IEEE Transactions on Vehicular Technology, vol. 18, no. 2, pp. 313-316, Feb. 2014.

[5] Z. Ding, Z. Yang, P. Fan, and H. V. Poor, "On the performance of non-orthogonal multiple access in 5G systems with randomly deployed users," IEEE Signal Processing Letters, vol. 21, no. 12, pp. 1501-1505, Dec. 2014.

[6] Y. Fu, Y. Chen, and C. W. Sung, "Distributed power control for the downlink of multi-cell NOMA systems," IEEE Transactions on Wireless Communications, vol. 16, no. 9, pp. 6207-6220, Sep. 2017.

[7] C. W. Sung and Y. Fu, "A game-theoretic analysis of uplink power control for a non-orthogonal multiple access system with two interfering cells," IEEE Vehicular Technology Conference (VTC), pp. 1-5, May. 2016.

[8] J. Zhao, Y. Liu, K. K. Chai, A. Nallanathan, Y. Chen, and Z. Han, "Resource allocation for non-orthogonal multiple access in heterogeneous networks," IEEE International Conference on Communications (ICC), pp. 1-6, May. 2017

[9] S. Luo and K. C. Teh, "Adaptive transmission for cooperative NOMA system with buffer-aided relaying," IEEE Communications letters, vol. 21, no. 4, pp. 937-940, 2017.

[10] J. Choi, "Non-orthogonal multiple access in downlink coordinated twopoint systems," IEEE Communications Letters, vol. 18, no. 2, pp. 313316, Feb. 2014.

[11] B. Wang, L. Dai, Z. Wang, N. Ge, and S. Zhou, "Spectrum and energyefficient beamspace MIMO-NOMA for millimeter-wave communications using Lens antenna array," IEEE Journal on Selected Areas in Communications, vol. 35, no. 10, pp. 2370-2382, Oct. 2017.

[12] Y. Fu, L. Salaün, C. W. Sung, and C. S. Chen, "Distributed power allocation for the downlink of a two-cell MISO-NOMA system," IEEE Vehicular Technology Conference (VTC), pp. 1-6, Jun. 2018.

[13] Z. Wei, J. Guo, D. W. K. Ng, and J. Yuan, "Fairness comparison of uplink NOMA and OMA," IEEE Vehicular Technology Conference (VTC), pp. 1-6, Mar. 2017.

[14] A. Maatouk, E. Caliskan, M. Assaad, M. Koca, G. Gui, and H. Sari, "Relaxing the condition of power imbalance in NOMA: Multiple access using two sets of orthogonal signal waveforms," to appear in proc. IEEE International Conference on Communications (ICC), pp. 1-6, May. 2018.

[15] — - "Frequency-domain NOMA with two sets of orthogonal signal waveforms," IEEE Communications letters, vol. 22, no. 5, pp. 906-909, May. 2018.

[16] G. Gui, H. Huang, Y. Song, and H. Sari, "Deep learning for an effective non-orthogonal multiple access scheme," IEEE Transactions on Vehicular Technology, Jun. 2018. [Online]. Available: DOI:10.1109/TVT.2018.2848294

[17] A. Benjebbour, A. Li, Y. Saito, Y. Kishiyama, A. Harada, and T. Nakamura, "System-level performance of downlink NOMA for future LTE enhancements," IEEE Global Telecommunications Conference (Globecom), pp. 66-70, Dec. 2013. 
[18] Y. Sun, D. W. K. Ng, Z. Ding, and R. Schober, "Optimal joint power and subcarrier allocation for MC-NOMA systems," IEEE Global Telecommunications Conference (Globecom), pp. 1-6, Dec. 2016.

[19] X. Li, C. Li, and Y. Jin, "Dynamic resource allocation for transmit power minimization in OFDM-based NOMA system," IEEE Communications Letters, vol. 20, no. 12, pp. 2558-2561, Dec. 2016.

[20] F. Fang, H. Zhang, and J. Cheng, "Energy-efficient resource allocation for downlink non-orthogonal multiple access network," IEEE Transactions on Communications, vol. 64, no. 9, pp. 3722-3732, Sep. 2016.

[21] Z. Wei, D. W. K. Ng, J. Yuan, and H.-M. Wang, "Optimal resource allocation for power-efficient MC-NOMA with imperfect channel state information," IEEE Transactions on Communications, vol. 65, no. 9, pp. 3944-3961, 2017.

[22] B. Di, L. Song, and Y. Li, "Sub-channel assignment, power allocation, and user scheduling for non-orthogonal multiple access networks," IEEE Transactions on Wireless Communications, vol. 15, no. 11, pp. 76867698, Nov. 2016.

[23] P. Parida and S. S. Das, "Power allocation in OFDM based NOMA systems: A DC programming approch," IEEE Globecom Workshops, pp. 1026-1031, Dec. 2015.

[24] H. Zhang, D. K. Zhang, W. X. Meng, and C. Li, "User pairing algorithm with SIC in non-orthogonal multiple access system," IEEE International Conference on Communications (ICC), pp. 1-6, May. 2016.

[25] L. Lei, D. Yuan, C. K. Ho, and S. Sun, "Power and channel allocation for non-orthogonal multiple access in 5G systems: tractability and computation," IEEE Transactions on Wireless Communications, vol. 15, no. 12, pp. 8580-8594, Dec. 2016.

[26] D. A. Eckhardt and P. Steenkiste, "Effort-limited fair (ELF) scheduling for wireless," IEEE International Conference on Computer Communications (Infocom), pp. 1-10, Dec. 2000.

[27] W. Li and Y. Pan, Resource Allocation in Next Generation Wireless Networks, 1st ed. New York: Nova Science Publisher, Inc., 2006, vol. 5 .

[28] D. Tse and P. Viswanath, Fundamentals of wireless communication. Cambridge University Press, 2005.

[29] M. J. Osborne, "Mathematical methods for economic theory," http://mjo. osborne.economics.utoronto.ca/index.php/tutorial/index/1/cvn/t.

[30] S. Boyd and L. Vandenberghe, Convex optimization, 1st ed. Cambridge University Press, 2004.

[31] P. H. Calamai and J. J. Moré, "Projected gradient methods for linearly constrained problems," Mathematical Programming, vol. 39, no. 1, pp. 93-116, 1987.

[32] Y. Chen and X. Ye, "Projection onto a simplex," arXiv preprint arXiv:1101.6081, 2011

[33] J. B. Rosen, "Existence and uniqueness of equilibrium points for concave n-person games," Econometrica, vol. 33, no. 3, pp. 520-534, 1965.

[34] K. W. Shum, K. K. Leung, and C. W. Sung, "Convergence of iterative waterfilling algorithm for Gaussian interference channels," IEEE Journal on Selected Areas in Communications, vol. 25, no. 6, pp. 1091-1100, Aug. 2007.

[35] I. L. Glicksberg, "A further generalization of the Kakutani fixed point theorem with application to Nash equilibrium points," National Academy of Sciences, vol. 38, pp. 170-174, 1952.

[36] K. Fan, "Fixed points and minimax theorems in locally convex topological linear spaces," National Academy of Sciences, vol. 38, pp. 121-126, 1952.

[37] G. Debreu, "A social equilibrium existence theorem," National Academy of Sciences, vol. 38, pp. 886-893, 1952.

[38] G. Scutari, D. P. Palomar, F. Facchinei, and J. S. Pang, "Convex optimization, game theory, and variational inequality theory," IEEE Signal Processing Magazine, vol. 27, no. 3, pp. 35-49, May 2010.

[39] T. M. Cover and J. A. Thomas, Elements of information theory. New York, Wiley, 1991.

[40] A. Zafar, M. Shaqfeh, M. S. Alouini, and H. Alnuweiri, "On multiple users scheduling using superposition coding over Rayleigh fading channels," IEEE Communication Letters, vol. 17, no. 4, pp. 733-736, Apr. 2013.

[41] GreenTouch, Mobile communications WG architecture doc2: Reference scenarios, May. 2013.

[42] X. Chen, A. Benjebbour, A. Li, and A. Harada, "Multi-user proportional fair scheduling for uplink non-orthogonal multiple access (NOMA)," IEEE Vehicular Technology Conference (VTC), pp. 1-5, May. 2014.

[43] Y. Saito, A. Benjebbour, Y. Kishiyama, and T. Nakamura, "Systemlevel performance evaluation of downlink non-orthogonal multiple access (NOMA)," Personal Indoor and Mobile Radio Communications (PIMRC), pp. 611-615, Sep. 2013.
[44] ETSI TR 136 942, LTE; Evolved Universal Terrestrial Radio Access (E-UTRA); Radio Frequency (RF) system scenarios, 3GPP TR 36.942 version 15.0.0 Release 15, June 2018.

[45] R. Jain, D. Chiu, and W. Hawe, A quantitative measure of fairness and discrimination for resource allocation in shared computer systems. Eastern Research Lab, Digital Equipment Corporation, DEC Technical Report 301, Sep. 1984. 